\title{
UNA NUEVA ESPECIE DE HOLMESINA SIMPSON (XENARTHRA, CINGULATA, PAMPATHERIIDAE) DEL PLEISTOCENO DE RONDÔNIA, SUDOESTE DE LA AMAZONIA, BRASIL
}

\author{
FLÁVIO GÓIS, GUSTAVO JUAN SCILLATO-YANÉ, \\ ALFREDO ARMANDO CARLINI \\ Departamento Científico Paleontología de Vertebrados, Facultad de Ciencias Naturales y Museo, \\ Universidad Nacional de La Plata, CONICET, Paseo del Bosque s/n, 1900, La Plata, Argentina. \\ goisf@fcnym.unlp.edu.ar,scillato@fcnym.unlp.edu.ar,acarlini@fcnym.unlp.edu.ar \\ MARTÍN UBILLA \\ Departamento de Evolución de Cuencas, Facultad de Ciencias, Iguá 4225, 11400, Montevideo, Uruguay. \\ martinubilla@gmail.com
}

\begin{abstract}
NEW SPECIES OF HOLMESINA SIMPSON (XENARTHRA, CINGULATA, PAMPATHERIIDAE) FROM THE PLEISTOCENE OF RONDÔNIA, SOUTHWEST AMAZONIA, BRAZIL. The Xenarthra Cingulata is one of the most characteristic groups in the Neotropical region. These American endemic mammals comprise several families, among them, the Pampatheriidae (middle Miocene-early Holocene) which are characterized by sharing traits with both the Dasypodidae and the Glyptodontidae. The genera Pampatherium Gervais \& Ameghino and Holmesina Simpson, occur in both North and South America. In North America, the species H. floridanus, H. septentrionalis and P. mexicanum are recorded from the late Pliocene to the Pleistocene, whereas H. occidentalis, H. paulacoutoi, H. majus, P. humboldtii and P. typum are recorded in the Pleistocene of South America. New specie of the genus Holmesina is here proposed based on a skull without mandible found at the locality Araras/Periquitos, right margin of Madeira River, municipality of Vila Nova Mamoré, Rondônia State, from the Rio Madeira Formation (late Pleistocene-Holocene). The new specie differs from the other species of the genera Holmesina and Pampatherium by a series of distinctive morphological and allometric features: markedly slender skull, narrow straight nasals, slightly convex frontals, long parietals, and little developed sagittal crest. The zygomatic arch long and slender, without the large lateral area at orbital level observed in the other species, infraorbital process absent; temporal fossa wide and excavated/ deep; supraoccipital very high, deep, with external occipital crest prominent and lambdoid crest expanded posteriorly; palate narrow, with imbrication molariforms between MF2, MF3 and MF4.
\end{abstract}

Key words: Rio Madeira Formation, Araras/Periquitos, Pleistocene, Rondônia, Holmesina.

RESUMO - Os Xenarthra Cingulata são um dos grupos mais característicos da Região Neotropical e são mamíferos endêmicos das Américas, que compreendem várias famílias. Entre elas, os Pampatheriidae (Mioceno médio-Holoceno inferior) caracterizamse por compartilhar caracteres com os Dasypodidae e Glyptodontidae. Os gêneros Pampatherium Gervais \& Ameghino e Holmesina Simpson encontram-se na América do Sul e América do Norte. Na América do Norte, entre o Plioceno superior e Pleistoceno estão registradas as espécies H. floridanus, H. septentrionalis e P. mexicanum. Para o Pleistoceno de América do Sul encontram-se H. occidentalis, H. paulacoutoi, H. majus, P. humboldtii e P. typum. Nova espécie para o gênero Holmesina é aqui proposta, baseada em um crânio sem mandíbula encontrado na localidade Araras/Periquitos, margem direita do rio Madeira, município de Vila Nova Mamoré, Rondônia, da Formação Rio Madeira (Pleistoceno superior-Holoceno). A nova espécie se diferencia das espécies de Holmesina e Pampatherium por possuir vários caracteres morfológicos e alométricos distintos: crânio menos robusto, nasais estreitos e retos, frontais suavemente convexos, parietais longos com crista sagital pouco marcada, arcada zigomática longa e delgada, sem a grande superfície lateral na altura da órbita presente nas outras espécies; processo infraorbitário ausente, fossa temporal ampla e escavada; supraoccipital bem alto, profundo, com uma crista occipital externa proeminente e lambdoidal expandida para trás; palato estreito e imbricação entre os molariformes MF2, MF3 e MF4.

Palavras-chave: Formação Rio Madeira, Araras/Periquitos, Pleistoceno, Rondônia, Holmesina.

\section{INTRODUCCIÓN}

Los Cingulata (Xenarthra) son uno de los grupos más característicos de la Región Neotropical y son mamíferos endémicos de las Américas. Comprenden dos clados principales: Glyptodontoidea y Dasypodoidea, que poseen como característica morfológica común la coraza dérmica (Carlini \& Scillato-Yané, 1993; McKenna \& Bell, 1997). 
La ubicación de los Pampatheriidae en uno u otro de estos clados es discutido, ya que poseen caracteres que los pueden asociar alternativamente a cualquiera de ellos. Así, según Patterson \& Pascual (1968, 1972), Paula Couto (1980), Engelmann (1985), Patterson et al. (1989), Carlini \& ScillatoYané (1993), Vizcaíno et al., (1998), De Iuliis et al. (2000), Hill (2005, 2006), Gaudin \& Wible (2006), Vizcaíno (2009) y Góis et al. (2010) son "gliptodontoides" por la geometría del dibujo de los osteodermos, la estructura de la coraza (con grandes escudos escapular y sobretodo el pélvico y menor número de bandas móviles), morfología de la región auditiva, cierta robustez craneana, forma y función del aparato masticatorio entre otras características; en tanto que para Simpson (1930), Hoffstetter (1958), Robertson (1976), Paula Couto (1979), Scillato-Yané (1982), Cartelle \& Bohórquez (1985) Abrantes \& Berqvist (2006) son “dasipodoideos", especialmente por tener la coraza dividida en tres áreas (escudo escapular, bandas móviles y escudo pélvico), la anatomía de los miembros y la morfología craneana con hocico largo.

Edmund (1985a, 1987), Downing \& White (1995), Edmund \& Theodor (1997) y De Iuliis \& Edmund (2002) consideran que los pampaterios son una familia, pero manifiestan que una revisión filogenética más completa es necesaria.

Los pampatéridos se registran con seguridad en Sudamérica desde el Mioceno medio (Edmund \& Theodor, 1997) hasta el Holoceno temprano (Cartelle \& Bohórquez, 1985; Cartelle, 1999; Scillato-Yané et al., 2005; Góis \& Scillato-Yané, 2008). Migraron hacia Norteamérica como parte del Gran Intercambio Biótico Americano (Marshall et al. 1984; Stehli \& Webb, 1985; Morgan \& Hulbert, 1995; Scillato et al. 1995; Carranza-Castañeda \& Miller, 2004; Flynn et al. 2005; Scillato-Yané et al. 2005; Carlini \& Zurita, 2010; Woodburne, 2010) y se diversifican allí durante el Plioceno tardío y el Pleistoceno tardío (Edmund, 1996; Carlini \& Zurita, 2010; Woodburne, 2010).

Para el Plioceno tardío y Pleistoceno se han reconocido dos géneros de Pampatheriidae, Holmesina Simpson, 1930 y Pampatherium Gervais \& Ameghino, 1880, de amplia distribución latitudinal, ya que sus registros se extienden desde la Provincia de Buenos Aires (Argentina) hasta México y Estados Unidos de América (Edmund, 1996; Scillato-Yané et al. 2005; Mead et al. 2007; Góis \& Scillato-Yané, 2008; Carlini \& Zurita, 2010).

Al igual que los gliptodontes, los pampatéridos han sido interpretados como formas básicamente herbívoras (Winge, 1941; Vizcaíno et al., 1998; De Iuliis et al., 2000; Vizcaíno, 2009).

En esta contribución damos a conocer una nueva especie de Holmesina del Pleistoceno de Rondônia, Sudoeste de la Amazonia, Brasil, proveniente de la Formación Rio Madeira (Figura 1A).

\section{HISTORIA NOMENCLATURAL, TAXONÓMICA Y FILOGENÉTICA}

El primer descubrimiento de restos de pampatéridos fue realizado por Lund (1839) en las cavernas de Lagoa Santa (Minas Gerais, Brasil), quien refirió los materiales a su nueva especie Clamydotherium humboldtii Lund, 1839. Sin embargo, Lund (1839) desconocía que el nombre genérico estaba previamente ocupado por una especie de gliptodonte descripta por Bronn (1838), lo que tornó inválida su nominación. En el mismo año, Öersted (1839) mencionó la especie de Lund (1839) en una breve nota, pero por un lapsus tipográfico modificó el nombre genérico de Chlamydotherium a Chlamytherium; además, la nota de Öersted (1839) apareció publicada unos meses antes que la de Lund.

De esta manera, y por un error, se introdujo el nuevo nombre genérico Chlamytherium de acuerdo con Cartelle \& Bohórquez (1985). Este error fue conservado por muchos autores (e.g. Winge, 1915; Castellanos, 1927, 1937; Hoffstetter, 1952; James, 1957).

Ameghino (1875) estableció el género Pampatherium, basado en la especie P. typus (sic). Es muy probable que Ameghino (1875), en esa época, desconociera por completo las especies pleistocenas de Brasil (Chlamydotherium humboldtii y C. majus Lund, 1842). La publicación original de Ameghino (1875) se perdió o bien nunca fue editada, hay dudas al respecto. Las posibilidades son dos: (i) a Alfredo J. Torcelli, director de la edición "Obras Completas y Correspondencia Científica de Florentino Ameghino" (nota al pie, vol. 2, p.13, nota 1, La Plata, 1914), no le fue posible encontrar ninguno de los escasos ejemplares supuestamente publicados en Mercedes en 1875. No obstante, Alfredo Torcelli publicó parte de los manuscritos originales truncos ("Obras completas", vol. 2, 1914, p. 13-17). En estos párrafos se menciona P. typus (sic), pero la página correspondiente a la supuesta edición original no pudo determinarse (ver Paula Couto, 1954; Mones, 1986), y (ii) no obstante, la alternativa más probable es la que indica Mones (1994), en el sentido de que esa obra temprana de Florentino Ameghino no fue publicada en 1875, sino recién en 1914.

Gervais \&Ameghino(1880) se aperciben que Pampatherium de Argentina es un sinónimo de Chlamydotherium del Brasil. Es la primera vez, aparentemente, que el nombre Pampatherium aparece édito; obviamente, sus autores son formalmente Gervais \& Ameghino (1880). Más adelante, tanto Chlamydotherium (o Chlamytherium) como Pampatherium continuaron usándose. Entre los autores que optaron por el primero (con su variante) están Ameghino (1883a,b), Leidy (1889b), Sellards (1915), Winge (1915), Castellanos (1927, 1937), Simpson (1930), Bordas (1939) y James (1957); en cambio, utilizan Pampatherium Paula Couto (1954, 1979, 1980), Scillato-Yané (1982), Cartelle \& Bohórquez (1985) y Edmund (1987, 1996).

En 1888, en Peace Creek (Florida, EUA), fueron exhumados los primeros restos (osteodermos) de pampatéridos en América del Norte, por parte del aficionado Joseph Wilcox. Leidy (1889a) describe dichos restos y los clasifica como Glyptodon septentrionalis Leidy, 1889. En el mismo año Leidy (1889b) reconoce que no se trata de un gliptodonte sino de un pampatérido relacionado con las especies de Brasil; en consecuencia, asigna este material a Chlamytherium humboldtii (= Pampatherium humboldtii), determinación que se mantuvo invariable hasta 1915. En ese año, Sellards (1915), estudia una 
mandíbula y osteodermos provenientes de Vero (Florida), y menciona asimismo materiales de otras localidades del estado de Florida. Los atribuye a C. septentrionale (sic), la determinación específica original de Leidy (1889a), es decir una especie distinta de las encontradas en Brasil. Posteriormente, Simpson (1930) propone el nuevo género Holmesina, de donde resulta la nueva combinación $H$. septentrionalis, especie tipo.

Ha tenido lugar una gran divergencia de opiniones en cuanto a la consideración de la jerarquía taxonómica de los pampatéridos: Bordas (1939) llega a atribuirles el estatus de superfamilia, Chlamydotherioidea; otros, como Simpson (1930), Hoffstetter (1952, 1953), Patterson (1967), Scillato-Yané (1982), Marshall et al. (1984), Cartelle \& Bohórquez (1985), Cartelle (1999) y Abrantes et al. (2006) los consideran como una subfamilia (Pampatheriinae). Para Abrantes \& Bergqvist (2006, p. 265) los pampaterios constituyen verdaderos dasipódidos, pero en una nueva tribu dentro de los Tolypeutinae. Autores como Ameghino (1883a,b), James (1957), Engelmann (1985), Edmund (1985b, 1987, 1996), Carlini \& Scillato-Yané (1993), Paula Couto (1980), Scillato-Yané et al. (1995), Edmund \& Theodor (1997), De Iuliis \& Edmund (2002), Scillato-Yané et al. (2005) y Carlini \& Zurita (2010), reconocieron el estatus de familia, el más aceptado en la actualidad.

A su vez, y en consideración de sus relaciones con los demás cingulados, Patterson \& Pascual (1968) removieron los pampaterios de los dasipódidos, ubicándolos en la base de los Glyptodontidae, según la idea implícita de que la presencia de bandas transversales móviles constituye un carácter primitivo dentro de los Cingulata.

Engelmann (1985) propuso, en su análisis filogenético, una dicotomía primaria dentro de los Cingulata e incluyó los Glyptodontidae junto con los Pampatheriidae y los armadillos Eutatini en un clado nominado Glyptodonta, cuyo grupo hermano son los Dasypoda. Según Engelmann (1985: 55), la principal sinapomorfia que reúne a los Glyptodonta es "The presence of a central island of compact dentine that is more resistant than the sourrounding dentine that comprise most of the tooth".

Más recientemente, Carlini \& Scillato-Yané (1993) colocan los Pampatheriidae como el grupo hermano de los Glyptodontidae. Contrariamente, Abrantes y Bergqvist (2006) proponen que los pampatéridos son verdaderos "armadillos" (Dasypodidae) y según sus resultados deben incluirse dentro de los Tolypeutinae (subfamilia que también reúne Priodontes y Cabassous), como grupo hermano de Tolypeutes.

Finalmente, los últimos análisis filogenéticos de Gaudin \& Wible (2006) y Porpino et al. (2009) proponen que los pampatéridos son el grupo hermano de los gliptodontes, como ya había sido propuesto por otros autores (e.g. Carlini \& Scillato-Yané, 1993).

\section{CONTEXTO GEOMORFOLÓGICO, GEOLÓGICO Y ESTRATIGRÁFICO}

El área de estudio abarca la porción sudoeste de la región Amazónica dentro de los límites del Estado de Rondônia, Cuenca del Abunã. En la columna del Cenozoico allí expuesto fue reconocida, en la base, la Formación Solimões (considerada entonces de antigüedad Plioceno tardíoPleistoceno temprano), seguida por sedimentos fluviales y fluvio-lacustres de edad Pleistoceno tardío, y por aluviones recientes (Radambrasil, 1978).

Posteriormente, Adamy \& Romanini (1990) y Adamy \& Pereira (1991), designaron como "Cobertura Cenozoica" el conjunto constituido por: (i) la Formación Solimões; (ii) la Formación Jaci-Paraná (o Formación Içá de Schobbenhaus, 1984) (Pleistoceno - Holoceno); y (iii) las lateritas y los sedimentos aluviales actuales.

Recientemente, Rizzotto (2005) separó la parte inferior de la Formación Jaci-Paraná (depósitos esencialmente fluviales), denominándola Formación Rio Madeira (Pleistoceno tardíoHoloceno), uniendo a ella lo que previamente se había considerado como Formación Solimões. La Formación JaciParaná quedó limitada entonces a los depósitos suprayacentes (Holoceno). Además, quedó descartada la presencia de la Formación Solimões en la Cuenca del Abunã.

Según Rizzotto et al. (2007) la Formación Rio Madeira (Figura 1B) comprende dos unidades principales. La unidad inferior está constituida por sedimentos arcillosos macizos a localmente laminados y bioturbados, que contienen detritus orgánicos representados por hojas carbonizadas y restos de fragmentos de vegetales. La unidad superior, de potencia más variable que la inferior, consta de sedimentos conglomerádicos, compuestos por clastos de cuarzo, matriz de arena media a gruesa, en general ferruginosa y endurecida por la cementación de óxidos e hidróxidos de hierro. De este nivel conglomerádico se exhumaron fósiles pleistocenos.

Se conocen tres localidades fosilíferas para la Formación Rio Madeira, asociadas a "garimpos" (zonas mineras) intensamente exploradas en las décadas del 1980 y 1990 del siglo XX. Las localidades están ubicadas en la margen derecha del río Madeira y son: (i) Araras/Periquitos, municipio de Vila Nova Mamoré (1003'01'S y 6519'31'O), localidad de la mayoría de los taxones registrados para el Estado, de aquí proviene el material tipo Holmesina n. sp.; (ii) Taquara, municipio de Porto Velho; y (iii) Prainha, Distrito de MutumParaná, municipio de Porto Velho (Figuras 1A-B).

De acuerdo a Rizzotto \& Oliveira (2005), dataciones ${ }^{14} \mathrm{C}$ indican una antigüedad pleistocena superior, entre los $27.310 \pm 200$ y $>46.310 \pm 200$ años AP. La primera datación debe ser considerada como la más probable para la edad de depositación de parte de la secuencia, en tanto que la segunda puede ser interpretada como la edad máxima de depositación. Estos autores, de acuerdo a análisis palinológicos, sostienen un contexto de bosque húmedo para estos depósitos.

Los primeros registros fósiles para la Formación Rio Madeira fueron citados por Adamy \& Pereira (1991) y atribuidos a Eremotherium Spillmann, 1948, y Stegomastodon Pohlig, 1912. Posteriormente, nuevos registros de mamíferos fueron mencionados por Sant'Anna Filho \& Vidal (1999), a saber: Mixotoxodon Van Frank, 1957, Toxodon Owen, 1837, Pampatherium (en realidad Holmesina, v. infra), Tapirus Brünnich, 1772, Inia d'Orbigny, 1834 y Trichechus Linnaeus, 1758. Nascimento et al. (2005) citan por primera vez 
Glyptodon Owen 1839 y Sclerocalyptus Ameghino 1891, indet. (actualmente Neosclerocalyptus Paula Couto 1957, ver Zurita, 2007; Zurita et al., 2007). Góis et al. (2004), Góis (2005) y Góis \& Scillato-Yané (2008) reconocen el género Holmesina entre los materiales recuperados. Porto et al. (2004) describe tres familias de Tardigrada:
Megatheriidae, Megalonychidae y Mylodontidae. Posteriormente, Holanda \& Cozzuol (2006) identifican un nuevo morfotipo de Tapirus para América del Sur, que posteriormente Holanda et al. (2011) describen como una nueva especie, Tapirus rondoniensis Holanda, Ribeiro \& Ferigolo, 2011.
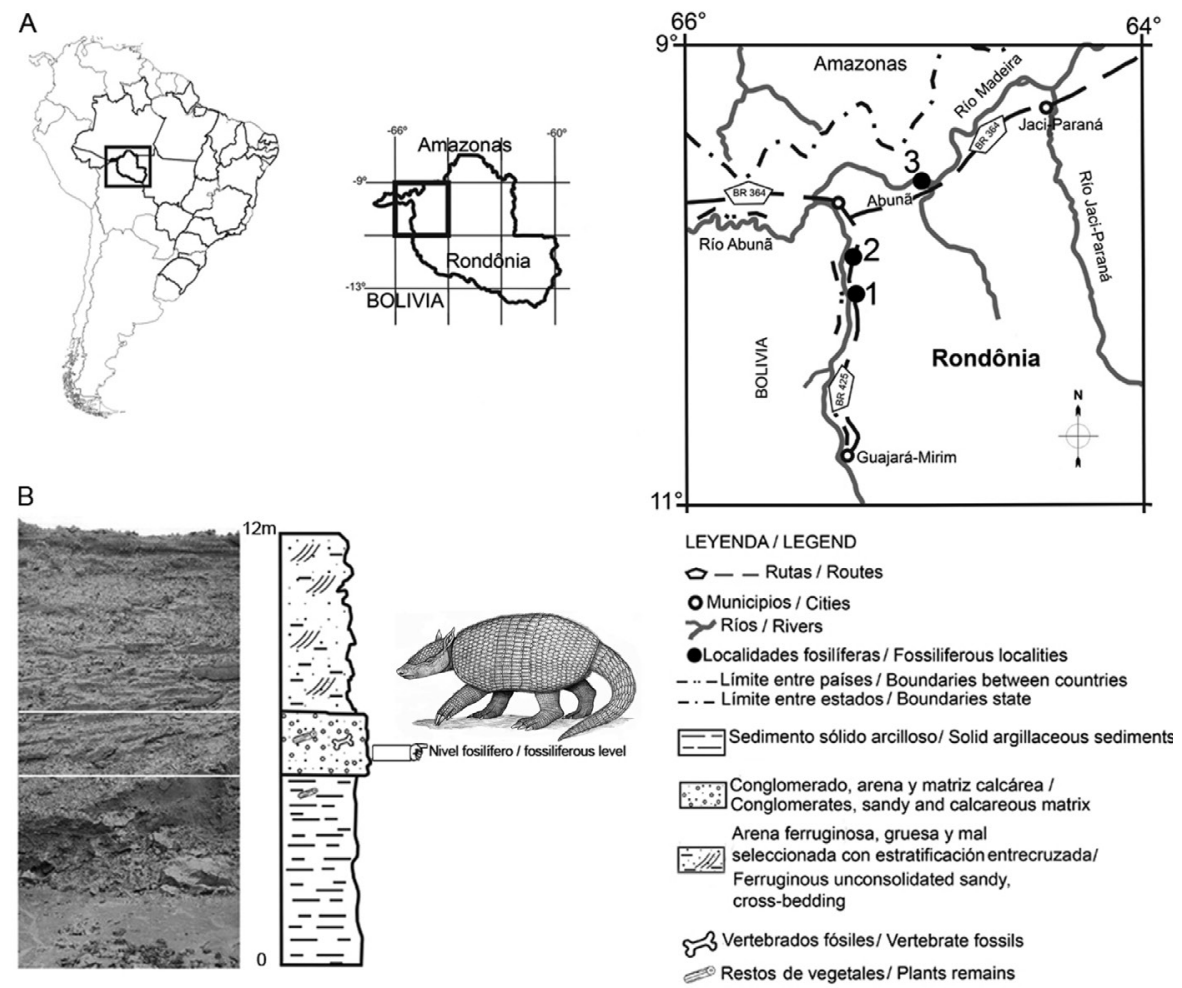

Figura 1. A, Mapa de localización del área de estudio y localidades fosilíferas en el Río Madeira, Estado de Rondônia, Brasil. 1, Araras/Periquitos, 2, Taquara y 3, Prainha. B, Sección tipo de la Formación Río Madeira, propuesta por Rizzotto et al., (2007) (modificado de Holanda et al., 2011).

Figure 1. A, Location map of the study area and fossiferous localities on the Madeira River, Rondônia state, Brazil. 1, Araras/Periquitos, 2 , Taquara y 3, Prainha. B, Type section of the Rio Madeira Formation, proposed by Rizzotto et al., (2007) (modified from Holanda et al., 2011).

\section{MATERIAL Y MÉTODOS}

El material estudiado se encuentra deposita en el Museu do Estado de Rondônia, Porto Velho, Rondônia, Brasil bajo las siglas MERO. Se trata de un cráneo sin mandíbula colectado en la localidad Araras/Periquitos, margen derecha del río Madeira, en el municipio de Vila Nova Mamoré, Rondônia, Formación del Río Madeira, cuya edad se atribuye al Pleistoceno tardío-Holoceno.

Se realizaron comparaciones con los materiales homólogos disponibles de las otras especies del géneros Pampatherium: P. humboldtii (MHD-P-28), P. typum (MACN Pv 11543); y Holmesina: H. septentrionalis (HMNS 173), H. occidentalis (ROM 3881), H. majus (UZM: 2314) y H. paulacoutoi (holotipo MCL-501/01) y aun de géneros emparentados: Scirrotherium hondaensis (holotipo UCMP 40201); Vassallia maxima (FMNH 14424) y Kraglievichia paranense (MACN Pv 2617, 8943). Con respecto a $S$. hondaensis fueron tratados solamente la morfología de algunos molariformes del maxilar, pues el cráneo (holotipo) está muy fragmentado.

Los caracteres craneanos utilizados en esta contribución fueron estudiados y analizados especialmente por Simpson (1930), Bordas (1939), James (1957), Cartelle \& Bohórquez (1985), Ubilla (1996), Edmund \& Theodor (1997), Vizcaíno et al. (1998), De Iuliis et al. (2000), De Iuliis \& Edmund (2002) y Góis \& Scillato-Yané (2008).

Abreviaturas institucionales. FMNH, Field Museum Natural History, Chicago, Estados Unidos de América; HMNS, Houston Museum of Natural Science, Houston, Texas, Estados Unidos de América; MACN, Museo Argentino de Ciencias Naturales "Bernardino Rivadavia", Buenos Aires, Argentina; MCL-PUC/MG, Museu de Ciências Biológicas da Pontifícia Universidade Católica de Minas Gerais, Belo Horizonte, Brasil; MERO, Museu do Estado de Rondônia, Porto Velho, Rondônia, Brasil; MHD-P, Museo Histórico Departamental de Artigas, Uruguay; MLP, División Paleontología Vertebrados, Facultad de Ciencias Naturales y Museo, Universidad Nacional de La Plata, Argentina; ROM, Royal Ontario Museum, Toronto, Canadá; UCMP, University of California, Museum of Paleontology, Estados Unidos de América; UZM, Universitets Zoologisk Museum, Copenhagen, Dinamarca. 
Abreviaturas utilizadas en las medidas craneanas. ABCO, ancho bicondilar; ALMC, ancho latero-medial del cóndilo; AMFM, ancho máximo del foramen magnum; APDP, ancho del paladar entre los dientes posteriores; LB, longitud basal; LCB; longitud condilobasal; LF; longitud frontal; $\mathbf{L N}$, longitud nasal; LP, longitud palatal; LPA, longitud parietal; LT, longitud total; LSDM, longitud de la serie dentaria maxilar, MF, molariformes.

\section{PALEONTOLOGÍA SISTEMÁTICA}

\author{
Superorden XENARTHRA Cope, 1889 \\ Orden CINGULATA Illiger, 1811 \\ Familia PAMPATHERIIDAE Paula Couto, 1954 \\ Holmesina Simpson, 1930
}

Especie tipo. Holmesina septentrionalis (Leidy, 1889) Simpson, 1930.

H. rondoniensis sp. nov.

(Figuras 2A-E; 3A)

Etimología. "rondoniensis", en homenaje a Cândido Mariano da Silva Rondon (1865-1958), de quien toma el nombre el Estado de Rondônia.

Holotipo. MERO-P-002, cráneo prácticamente completo en buenas condiciones de conservación, con la región palatal y rostral completas, con solo dos molariformes preservados, ambos de la serie dentaria derecha, el MF3 y MF8 rotos en la parte distal; el resto de los molariformes está ausente, pero se conservan sus alvéolos. Las apófisis pterigoideas en su parte distal están deterioradas. La región frontal está en gran parte fragmentada, pero se conserva en su totalidad la porción derecha con la cresta frontal externa y la porción anterior; hacía adelante y en ambos lados, persisten regiones importantes como los límites: fronto-maxilar, naso-frontal, maxilo-lagrimal, fronto-lagrimal y dorsalmente la sutura nasofrontal y fronto-parietal. Falta el arco zigomático derecho y se conserva por completo la región basioccipital y ótica.

Paratipo. El holotipo solamente.

Procedencia geográfica y estratigráfica. Araras/Periquitos, Municipio Vila Nova Mamoré (Rondônia, Brasil); Formación Rio Madeira, Pleistoceno tardío-Holoceno (Figuras 1A-B).

Diagnosis. Cráneo más grácil y estilizado que en las restantes especies de Holmesina (H. septentrionalis, H. occidentalis, $H$. majus y $H$. paulacoutoi), mucho más que en las de Pampatherium (P. typum y P. humboldtii) y en Vassalia maxima, gracilidad similar a la de Kraglievichia paranense. Rostro más estrecho y acuminado, sobre todo en la porción más anterior, patrón ausente en las demás especies del género y notablemente diferente de las de Pampatherium. Región nasal más corta que en las restantes especies de Holmesina y mucho más que en las de Pampatherium. Nasales con el ancho anterior mayor que el posterior, al igual que en $V$. maxima y $K$. paranense (anchos equivalentes en las otras especies de Holmesina, y el anterior mucho mayor que el posterior en Pampatherium). Frontales más pequeños que en las restantes especies de Holmesina y que en Pampatherium; poco convexos dorsal y lateralmente, a diferencia de $V$. maxima (muy convexos), las restantes especies de Holmesina (regularmente convexos), K. paranense (menos convexos que en $H$. rondoniensis) y Pampatherium (frontales menos convexos todavía). Órbita más alargada que en todas especies comparadas. Región parietal, larga, alta y menos rugosa que en Pampatherium y en $V$. maxima, con pocos canales y forámenes vasculares; cresta sagital muy estrecha y elevada (pero menos que en $K$. paranense y mucho menos que en $V$. maxima). Arcada cigomática larga y menos robusta que en $P$. typum, V. maxima, H. majus, $H$. occidentalis, $H$. paulacouto $i$ y H. septentrionalis (en orden decreciente de robustez). En el punto de contacto de los huesos yugal-escamosal, la arcada cigomática de $H$. rondoniensis es menos extendida dorsoventralmente que en todas las especies anteriormente citadas. Sin procesos postorbitarios e infraorbitario (presentes en los restantes Pampatheriidae). Espacios interalveolares más reducidos que en $S$. hondaensis, $V$. maxima, las restantes especies de Holmesina y Pampatherium, pero menos que K.paranense. MF1 muy próximo al margen inferior de las narinas; MF2, MF3 y MF4 imbricados entre sí; MF5 reniforme (en las restantes especies del género bilobulado). Solamente MF6, MF7 y MF8 francamente bilobulados; MF9 oblongo, con un leve surco longitudinal labial. Sin tercer lóbulo (intermedio), a diferencia de $K$. paranense (MF6 y MF7), H. occidentalis (MF8), H. paulacoutoi (MF5 y MF8), H. majus (MF6 y MF7) y H. septentrionalis (MF7), P. typum y $P$. humboldtii (ambos a partir del MF5 hacia atrás) y $V$. maxima (a partir del MF6). Paladar más estrecho (anterior y posteriormente) que en las restantes especies de Holmesina y mucho más que en Pampatherium, pero más ancho que en $V$. maxima y $K$. paranense. Apófisis pterigoides poco rugosas, delgadas y apenas expandidas lateralmente (rugosas, robustas y regularmente expandidas en los restantes Pampatheriidae). Región occipital muy alta, estrecha, con la cresta occipital pronunciada y dos fosas profundas, una a cada lado de ella, a diferencia de los restantes Pampatheriidae.

Diagnosis. Skull more graceful and stylish than in the other species Holmesina (H. septentrionalis, H. occidentalis, $\mathrm{H}$. and $\mathrm{H}$. paulacoutoi majus), much more than in the Pampatherium (P. typum. and P. humboldtii) and Vassalia maxima, similar to gracilidad Kraglievichia paranense. Acuminate narrowest face and, especially in the anterior portion, pattern absent in the other species of the genus and notably different from those of Pampatherium. Nasal region shorter than in the other species of Holmesina and more than in the Pampatherium. Anterior nasal width greater than the posterior, as in $\mathrm{V}$. maxima and $\mathrm{K}$. paranense (equivalent widths in the other species of Holmesina, and much higher than the previous post in Pampatherium). Front smaller than in the other species and in Pampatherium and Holmesina; slightly convex dorsal and laterally, unlike V. maxima (very convex), the remaining species Holmesina (regularly convex), K. paranense (less convex than in $\mathrm{H}$. rondoniensis) and Pampatherium (less convex front yet). Elongated orbit in all species compared. Parietal region, long, high and less 

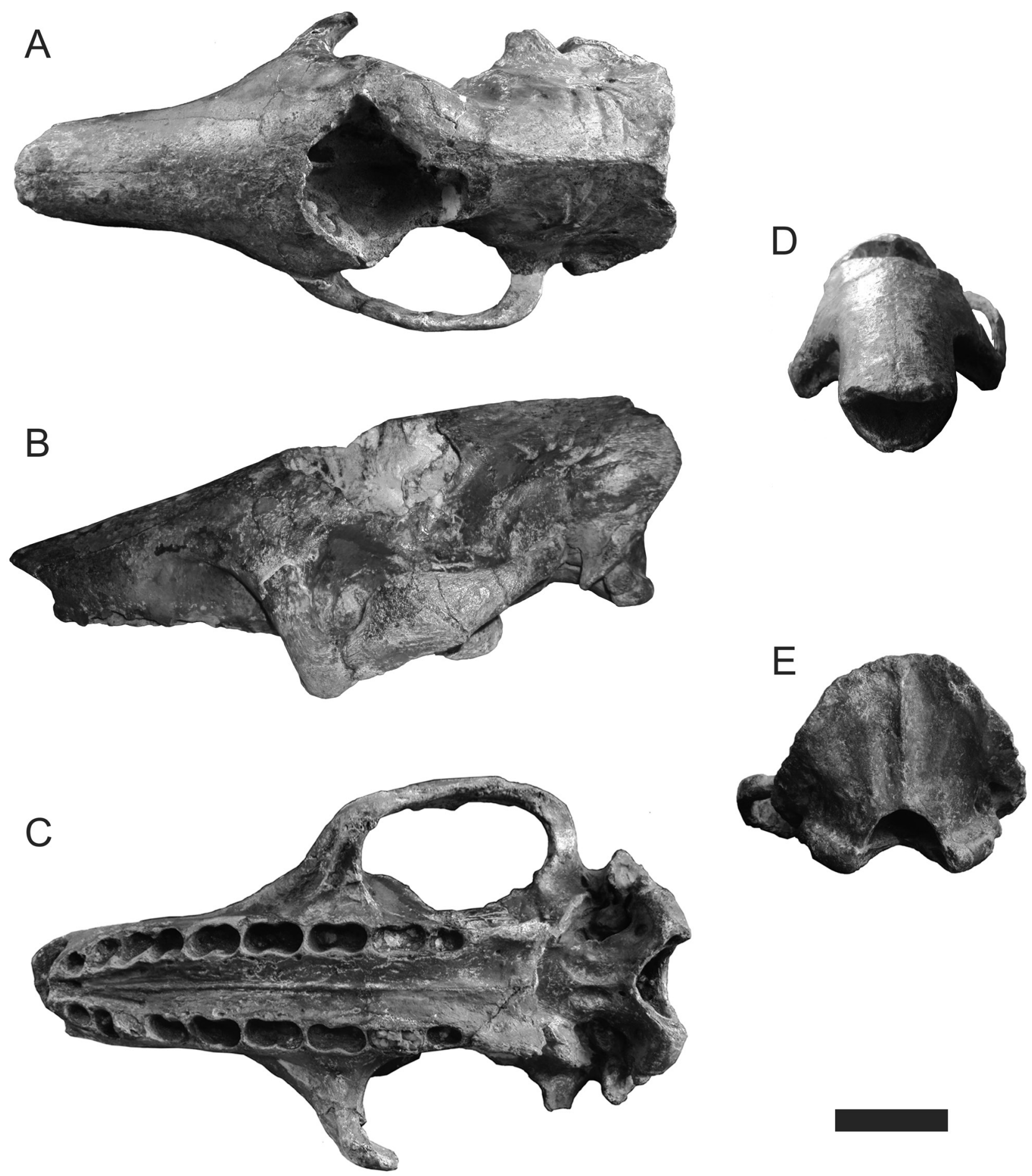

Figura 2. Cráneo de Holmesina rondoniensis sp. nov., MERO-P-002. A, vista dorsal; B, lateral; C, ventral; D, anterior; $\mathbf{E}$, posterior. Escala = $50 \mathrm{~mm}$.

Figure 2. Skull of Holmesina rondoniensis sp. nov., MERO-P-002. A, lateral; B, lateral; C, ventral, D, anterior; E, posterior views. Scale bar = $50 \mathrm{~mm}$.

rough than Pampatherium and V. maxima, with few channels and vascular foramina; very narrow sagittal crest high (but less than in $\mathrm{K}$. paranense much less than in V. maxima). Zygomatic arch longer and less robust than in $\mathrm{P}$. typum, V. maxima, H. majus, H. occidentalis, H. paulacoutoi and $H$. septentrionalis (in decreasing order of strength). At the point of contact-escamosal jugal bones, the zygomatic arch of $\mathrm{H}$. rondoniensis dorsoventrally is less widespread in all the above species. No postorbital processes and infraorbital (present in the remaining Pampatheriidae). Interalveolar spaces smaller than in Scirrotherium hondaensis, V. maxima, the remaining species and Pampatherium, Holmesina but less than $\mathrm{K}$. paranense. MF1 very close to the bottom of the nostrils; $M F 2, M F 3$ and MF4 overlapping each other; MF5 reniform (in the remaining species of bilobed). Only MF6, MF8 MF7 and frankly bilobed; MF9 oblong, with a slight longitudinal 
groove lip. No third lobe (intermediate), unlike K. paranense (MF6 and MF7), H. occidentalis (MF8), H. paulacoutoi (MF5 and MF8), H. majus (MF6 and MF7) and H. septentrionalis (MF7), P. typum and P. humboldtii (both from back MF5) and V. maxima (from MF6). Narrower palate (anterior and posterior) in the remaining Holmesina species and more than Pampatherium but wider than V. maxima and $\mathrm{K}$. paranense. Pterygoid little rough, thin and barely expanded laterally (rugged, robust and regularly expanded in the remaining Pampatheriidae). Occipital region very high, narrow, steep and occipital crest two deep trenches, one on each side of it, unlike the other Pampatheriidae.

Descripción y comparación. Vista dorsal: El cráneo de Holmesina rondoniensis posee una longitud total de 325 $\mathrm{mm}$, medidos con el paladar orientado horizontalmente (ver las restantes medidas comparadas en la Tabla 1). Los nasales miden $140 \mathrm{~mm}$ de largo, $5.1 \mathrm{~mm}$ de ancho proximal y 4.5 $\mathrm{mm}$ de ancho distal; son estilizados y estrechos. Distalmente, sobrepasan la porción anterior de los premaxilares y proximalmente contactan con los frontales por medio de una sutura sinuosa. El ancho distal es algo menor que el proximal, a diferencia de las restantes especies de Holmesina. El único ejemplar craneano de $H$. paulacoutoi (el holotipo), presenta la porción más posterior del rostro fragmentada, pero, de acuerdo a la región preservada, con toda probabilidad sería más ensanchada que en todas las especies de Holmesina; la porción anterior del rostro está ausente. $H$. occidentalis posee el ancho distal y proximal mayores que $H$. septenrionalis y $H$. majus. En Pampatherium humboldtii y P. typum los nasales se ensanchan notablemente hacia delante; en estas dos especies los márgenes de la narina correspondientes a dichos huesos conforman un reborde ancho, ausente en $H$. rondoniensis sp. nov. o en cualquier otra especie del género (Figuras

Tabla 1. Medidas craneanas $(\mathrm{mm})$ de $\mathbf{A}, H$. rondoniensis sp. nov., MERO-P-002; B, H. occidentalis, ROM 3881; C, H. majus, UZM 2314; D, H. paulacoutoi, MCL-501/01; E, H. septentrionalis, HMNS 173; F, Pampatherium typum, MACN Pv 11543; G, P. humboldtii, MHD-P-28. Abreviaturas: en el texto.

Table 1. Cranial measurements $(\mathrm{mm})$ of $\mathbf{A}$, Holmesina rondoniensis sp. nov., MERO-P-002; B, H. occidentalis, ROM 3881; C, H. majus, UZM 2314; D, H. paulacoutoi, MCL-501/01; E, H. septentrionalis, HMNS 173; F, Pampatherium typum, MACN Pv 11543; G, P. humboldtii, MHD-P-28. Abbreviations: see text.

\begin{tabular}{cccccccc}
\hline & A & B & C & D & E & F & G \\
\hline LT & 325 & 345 & 315 & 282 & 290 & 350 & 360 \\
LN & 145 & 162 & 156 & - & - & 164 & 174.6 \\
LF & 97 & 106 & 99 & - & - & 111 & 113.7 \\
LPA & 83 & 72 & 76 & 71 & - & 75 & 72 \\
LB & 269 & 283 & - & - & - & 304 & 285 \\
LCB & 291 & 302 & 270 & - & - & 325 & 340 \\
LP & 220 & 230 & - & 202 & 220 & 235 & 237 \\
APDP & 27 & 34 & 35 & 30 & 40 & 52 & 27.6 \\
LSDM & 180 & 190 & 197 & 180 & 165 & 200 & 195 \\
ALMC & 29 & 32 & 33.5 & 32 & 27 & 15 & 24.4 \\
ABCO & 88 & 86 & 85.4 & 90 & 70 & 70 & 83.5 \\
AMFM & 39 & 35 & 32 & 32 & 25 & 26 & 34 \\
\hline
\end{tabular}

3A-B). En general, los frontales de los Pampatheriidae son muy convexos. La región frontal de $H$. rondoniensis sp. nov. está en gran parte fragmentada, pero se conserva en su totalidad la porción derecha del frontal, con la cresta frontal externa y la sutura fronto-parietal; además, se conservan regiones importantes, o sea los límites de distintos huesos (e.g. suturas fronto-maxilar, naso-frontal, maxilo-lagrimal, fronto-lagrimal en posiciones dorsal y lateral). Parte de la cresta frontal izquierda (porción anterior) se conserva por completo, así como en la parte derecha las suturas frontonasal y fronto-parietal. Por las suturas fronto-nasal y frontoparietal que se conservan se entiende que los frontales eran más cortos que en cualquier otra especie pleistocena. Las suturas fronto-maxilar o maxilo-frontal, maxilo-lagrimal y fronto lagrimal que se mantienen preservadas demuestran claramente que, si los frontales estuvieran completos, tal región (dorsalmente) sería menos convexa que la de las otras especies del género. En síntesis, tiene el siguiente orden de robustez de los frontales: Vassalia maxima, H. occidentalis, H. septentrionalis, H. majus, H. rondoniensis, Kraglievichia paranense, P. typum y P. humboldtii. En el límite frontoparietal, el techo craneano de $H$. rondoniensis sp. nov. presenta una pequeña región plana que se extiende a ambos lados del plano sagital. Los parietales son más largos y estrechos que en $H$. occidentalis y H. paulacoutoi; la cresta sagital es muy estrecha y alta, más que la de Holmesina y menos que las de $K$. paranense y $V$. maxima. En la región inferior de los parietales de $H$. rondoniensis sp. nov., la fosa retro-cigomática es más profunda que en las restantes especies del género y que en Pampatherium; en H. rondoniensis sp. nov., los forámenes y canales vasculares están menos marcados. Entre los frontales se encuentra (latero-ventralmente) el proceso postorbitario, generalmente bien pronunciado y puntiagudo, presente en todas las especies hasta hoy reconocidas de Holmesina y Pampatherium, así como en $V$. maxima y $K$. paranense, pero en $H$. rondoniensis sp. nov. es ausente. La cresta lambdoidea en $H$. rondoniensis sp. nov. es pronunciada, poco rugosa y delgada; en la región mesial, la cresta lambdoidea forma una pequeña convexidad anterior; hacia ambos lados, se extiende a modo de sendas concavidades posteriores muy marcadas. En las otras especies: H. majus, H. occidentalis, $H$. septentrionalis y $H$. paulacoutoi dichas convexidades están menos proyectadas hacía atrás. H. paulacoutoi posee las convexidades menos acentuadas del género. No obstante, en las especies anteriormente citadas, presentan en la parte dorsal y lateral de la cresta lambdoidea un espesor considerable, sobretodo H. paulacoutoi. En P. humboldtii y P. typum dicha cresta es gruesa y muy rugosa con la convexidad anterior más marcada que las demás especies de Holmesina y mucho más que la de $H$. rondoniensis sp. nov. Las convexidades posteriores de $P$. humboldtii y $P$. typum son menos expandidas hacía atrás que en $H$. majus, H. occidentalis, H. septentrionalis y $H$. paulacoutoi en y mucho menos que $H$. rondoniensis sp. nov. La morfología de la cresta lambdoidea es un carácter distintivo entre los géneros Pampatherium y Holmesina. En Pampatherium dicha cresta (dorsal y lateralmente) es más homogénea y gruesa en toda su extensión, con muchas 


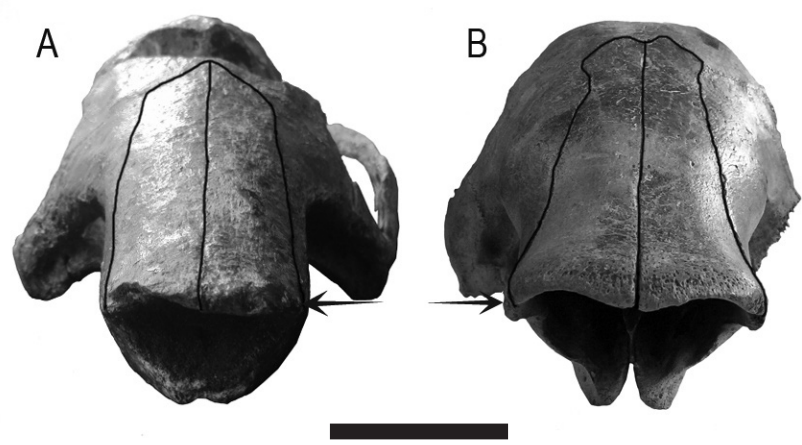

Figura 3. Comparación de los cráneos en vista frontal: A, Holmesina rondoniensis sp. nov., MERO-P-002; B, Pampatherium humboldtii, MHD-P-28. Nasales resaltados. La flecha indica el ensanchamiento máximo anterior. Escala $=50 \mathrm{~mm}$.

Figure 3. Comparative of frontal view of species: A, Holmesina rondoniensis sp. nov., MERO-P-002; B, Pampatherium humboldtii, MHD-P-28. Nasals marked. The arrow indicates the maximun anterior widening. Scale bar $=50 \mathrm{~mm}$.

rugosidades posiblemente relacionada a un mayor incremento de la musculatura masticatoria, mientras que las de Holmesina son mucho más gráciles y delgadas con pocas rugosidades, lo que implicaría un menor desarrollo muscular, con excepción de H. paulacoutoi (ver Vizcaíno et al., 1998; De Iuliis et al., 2000; De Iuliis \& Edmund, 2000). Con respecto a la cresta lambdoidea, $K$. paranense es más parecida a Holmesina y $V$. maxima a Pampatherium, lo cual es interesante desde un punto de vista evolutivo, pues ese carácter (junto con otros ya mencionados) parece reforzar aún más la relación de parentesco Kraglievichia-Holmesina por un lado y VassalliaPampatherium por el otro. Estudios morfofuncionales enfatizan la relación de Vassallia con Pampatherium (ver Vizcaíno et al., 1998; Góis et al., 2010).

Vista lateral: en la región más anterior del rostro, Holmesina rondoniensis sp. nov. presenta una cresta maxilar menos marcada y más baja que en las otras especies del género. En Pampatherium typum y P. humboldtii dicha cresta es muy pronunciada y casi llega a contactar los nasales. El foramen infraorbitario se halla en una posición más baja y posterior que en otros pampatéridos, a la altura del MF7. La fosa orbitaria de $H$. rondoniensis sp. nov. tiene límites poco definidos, salvo hacia adelante. No hay apófisis postorbitaria; en cambio, es relativamente pequeña y subcircular en $H$. majus, H. occidentalis y P. typum. A su vez, la fosas orbitarias de $H$. paulacoutoi, $H$. septentrionalis y $P$. humboldtii son mayores que las de $H$. majus, $H$. occidentalis y P. typum. En $H$. rondoniensis sp. nov. la arcada cigomática, en la zona de la sutura yugal-escamosal, es mucho menos extendida dorsoventralmente que en las restantes especies de Holmesina, así como en todas las de Pampatherium (en P. humboldtii la arcada no se conserva) y menor que Vassalia maxima, el único cráneo casi completo de Kraglievichia paranense no se conserva la arcada cigomática. No solamente es menos elevada la región mediana del arco en $H$. rondoniensis, sino que toda la arcada cigomática es más grácil y más larga que en los taxones mencionados; asimismo, el proceso infraorbitario está ausente.
Vista ventral: el paladar mide $220 \mathrm{~mm}$ y corresponde al $67 \%$ de la longitud total del cráneo. Es muy estrecho a nivel de los MF1 al MF3 y divergente hacia atrás hasta el MF5; los bordes alveolares son paralelos desde el MF4 hasta el MF9. En Holmesina y Kraglievichia paranense, el paladar es muy estrecho anteriormente (entre los molariformes), a saber: $K$. paranense (entre MF1-MF2 derechos e izquierdos); $H$. rondoniensis sp. nov. (MF1-MF3); H. occidentalis (MF1-MF2); H. majus (parte anterior del paladar ausente); H. paulacoutoi (MF1-MF4) y H. septentrionalis (MF1MF2). Contrariamente, Vassalia maxima y las especies de Pampatherium (P. humboldtii y P.typum) presentan un patrón morfológico diferente, aunque también con el paladar más estrecho anteriormente; las series de molariformes están más distanciadas y son paralelas entre sí. En cambio, en las especies de Holmesina, en K. paranense y en Scirrotherium hondaensis, los molariformes anteriores convergen imbricándose. En $V$. maxima, el paladar es más estrecho hasta los MF3, mientras que en $P$. typum y $P$. humboldtii lo son hasta los MF4. En estas tres especies, los bordes alveolares de los molariformes posteriores dibujan un amplio arco. En las especies de Holmesina y en $K$. paranense, después de la parte imbricada de la serie, los bordes alveolares sí divergen una sola vez, manteniéndose paralelos. La abertura de los orificios nasales externos de $H$. rondoniensis sp. nov. tiene forma de trapecio de contornos redondeados; es más pequeño, que en las otras especies del género, mucho más que en Pampatherium y algo semejante a $K$. paranense y $V$. maxima. El extremo anterior del paladar, constituido por los premaxilares, es de contorno semilunar, en cambio las demás especies de Holmesina poseen una pequeño prolongamiento hacia adelante, pero menor que las de Pampatherium (especialmente marcada en P. humboldtii). El primer molariforme de H. rondoniensis sp. nov. es muy próximo $(0.8 \mathrm{~mm})$ al margen anterior del paladar; también es muy próximo en $V$. maxima. En las restantes especies de Holmesina y en Pampatherium esta distancia es proporcionalmente el doble. Las apófisis pterigoides de $H$. rondoniensis sp.nov. son delgadas y poco rugosas; prosiguen la dirección de la serie dentaria. En $V$. maxima, $H$. paulacoutoi y H. occidentalis, dichas apófisis son mucho más gruesas y rugosas, y se expanden lateralmente por afuera de la dirección de la serie dentaria. El basioccipital de $H$. rondoniensis sp. nov. es muy alargado, más que en cualquier otra especie de Holmesina. En su región central se encuentran dos fosas muy estrechas y profundas separadas por una cresta fuerte y alta, que, presta inserción a los músculos rectos ventrales. En Pampatherium el basioccipital es más corto que $H$. rondoniensis sp. nov., pero son más anchas y con una cresta menos marcada las fosas citadas. En $V$. maxima y $K$. paranensis el basioccipital es proporcionalmente más grande que en $H$. rondoniensis y mucho más que en Pampatherium. Las fosas y crestas son menos marcadas que en las especies pleistocenas. La bula timpánica en los pampatéridos en general es muy pequeña y posee una forma globosa y de base semioval, pero el tamaño y el ancho difieren según los taxones. En $H$. rondoniensis sp. nov. es muy convexa y ancha, al igual que en $H$. septentrionalis. En $H$. paulacoutoi es de tamaño 
Holmesina

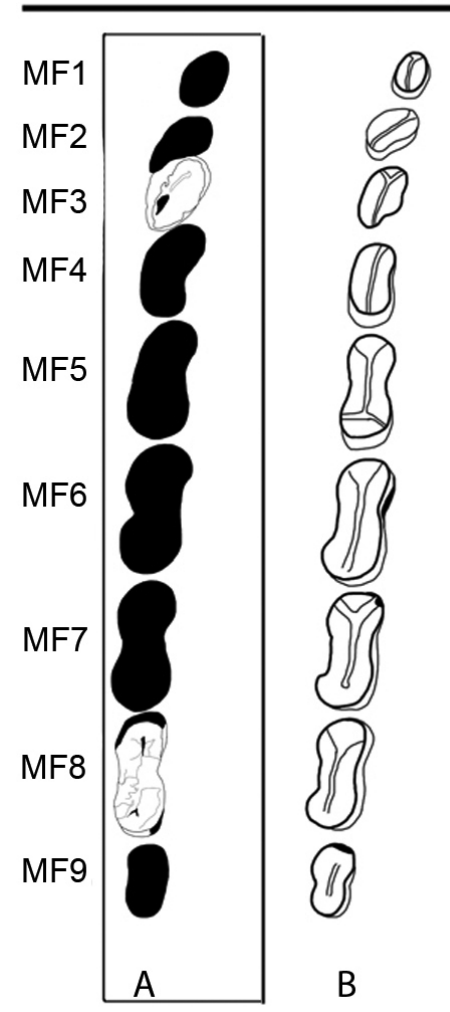

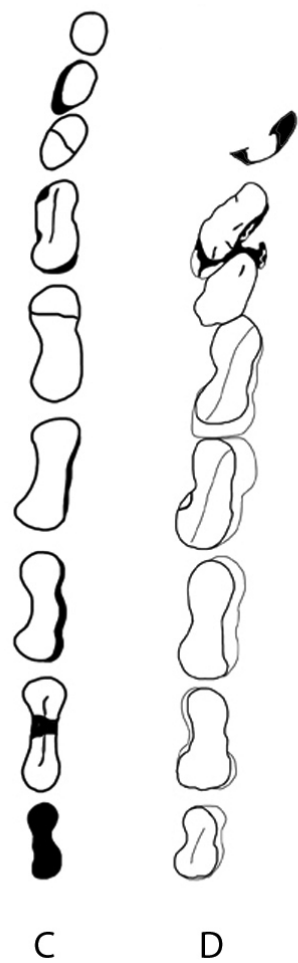

Pampatherium

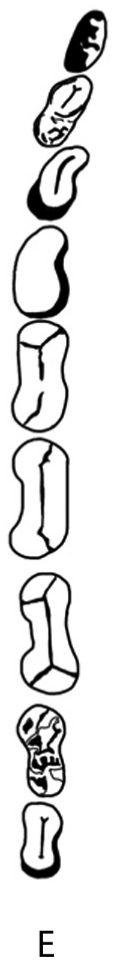

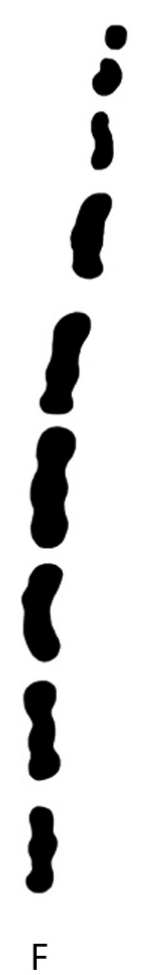

Figura 4. Serie dentaria superior derecha: A, Holmesina rondoniensis sp. nov., MERO-P-002; B, H. occidentalis, ROM 3881; C, H. majus, UZM 2314; D, H. paulacoutoi, MCL-501/01; E, H. septentrionalis, HMNS 173; F, Pampatherium typum, MACN Pv 11543; G, P. humboldtii, MHD-P-28. En negro, molariformes ausentes (forma inferida por el alvéolo); en algunos casos la superficie oclusal está total o parcialmente destruida. Algunas ilustraciones fueron completadas con la serie dentaria izquierda del mismo ejemplar. Ver dimensiones en Tabla 2. Sin escala.

Figure 4. Upper right tooth rows: A, Holmesina rondoniensis sp. nov., MERO-P-002; B, H. occidentalis, ROM 3881; C, H. majus, UZM 2314; D, H. paulacoutoi, MCL-501/01; E, H. septentrionalis, HMNS 173; F, Pampatherium typum, MACN Pv 11543; G, P. humboldtii, MHD-P-28. In black, lacking molariforms (inferred form based on the alveolus); in some cases the occlusal surface is completely or partially destroyed. Some illustrations were completed with the left dental series of the same specimen. See dimmensions in Table 2. Not to scale.

similar a $H$. rondoniensis sp. nov., pero algo más angosta, mientras que en $H$. occidentalis es muy pequeña y angosta. En P. typum y P. humboldtii las bulas son similares a las de $H$. occidentalis. Los cóndilos occipitales de H. rondoniensis sp. nov. posee un formato de paralelogramo de contornos redondeados; el diámetro lateromedial de cada uno de los cóndilos es un tercio menor que el diámetro transverso del foramen magnum, al igual que en $H$. occidentalis, H. majus y P. humboldtii. En H. paulacoutoi y H. septentrionalis ambas medidas son equivalentes. $P$. typum posee cóndilos occipitales muy pequeños y de forma subcuadrada; el diámetro lateromedial de ellos es dos tercios menor que el diámetro transverso del foramen magnum. Dorsalmente a los cóndilos se sitúa la fosa condiloide, muy profunda en $H$. rondoniensis sp. nov. y poco marcada en las otras especies de Holmesina. El foramen magnum es suboval, con el diámetro transverso muy largo es un tercio más grande que el anteroposterior y una pronunciada escotadura mediana dorsal. Esta escotadura es mucho menos pronunciada en $H$. occidentalis y en $P$. typum. En $P$. humboldtii el borde superior del foramen magnum forma un ángulo de $80^{\circ}$, notablemente acusado.

Vista occipital: Holmesina rondoniensis sp. nov. posee el supraoccipital alto, estrecho y escavado a ambos lados de la cresta occipital (Figura 2E). Dicha cresta es muy prominente; se extiende desde la parte superior de la cresta lambdoidea hasta el borde superior del foramen magnum. En H. occidentalis el supraoccipital es más bajo y poco excavado con la cresta menos elevada que en $H$.rondoniensis sp. nov. mientras $H$. paulacoutoi y $P$. humboldtii presentan un supraoccipital más excavado superiormente a la porción mediana de la cresta occipital. En $H$. majus y $P$. typum poseen un supraoccipital más bajo, de contorno semicircular y con la cresta occipital menos marcada.

Molariformes: el holotipo de Holmesina rondoniensis sp. nov. conserva solamente el MF3 y el MF8 derechos, en parte fragmentados; no obstante, tratándose de dientes euhipsodontes, la morfología y tamaño general puede inferirse de aquella de sus respectivos alvéolos. En la región anterior de la serie dentaria, Holmesina presenta los primeros molariformes imbricados, en tanto que en Pampatherium se disponen sin imbricación (Figura 4). En H. rondoniensis sp. nov. la imbricación se observa en los molariforme MF3 y alveolos del MF2 y MF4 (Figura 4A). De todas las especies del género, $H$. rondoniensis sp. nov. es la que presenta menores espacios interdentarios. En cuanto a $H$. paulacoutoi, Cartelle \& Bohórquez (1985, p. 236) comentan: "comparando 
com as outras espécies afins, H. paulacoutoi destaca-se num particular: o espaço interdentário. Todos os dentes situam-se muito próximos entre si". No obstante, en $H$. rondoniensis sp. nov. dichos espacios son aún menores, sobre todo en la región anterior de la series. Los valores obtenidos para la sumatoria de los respectivos espacios interdentarios en orden creciente son: $H$. rondoniensis sp. nov. $(6.4 \mathrm{~mm})$; H. paulacoutoi $(7.4 \mathrm{~mm}) ; H$. occidentalis $(14 \mathrm{~mm}) ; H$. septentrionalis (17 mm); H. majus (22,5 mm); P. typum (22, $5 \mathrm{~mm})$ y $P$. humboldtii $(24.4 \mathrm{~mm})$. Los alvéolos aumentan de tamaño de adelante hacia atrás hasta el MF6 (de mayor tamaño) y disminuyen progresivamente, característica general de todos los Pampatheriidae y en muchos otros Cingulata (Tabla 2). El alvéolo de MF1 es subcircular o circular, condición de todos los Pampatheriidae. Es el más pequeño de la serie superior; el alvéolo está inserto en el premaxilar, condición común a todos los pampaterios conocidos (De Iuliis \& Edmund, 2002). En H. rondoniensis sp. nov., los MF2 y MF3 tienen dimensiones similares y la cara labial plana o ligeramente cóncava. El alvéolo de MF2 posee además una ligera cresta lingual, indicando que el diente tiene un surco muy similar al de Kraglievichia paranense; en Scirrotherium hondaensis este surco es proporcionalmente más superficial y su ubicación más caudal. En $H$. paulacoutoi dicho surco es más ancho, similar al de $H$. occidentalis. Los MF2 de $H$. majus y $H$. septentrionalis que son subovalados y carecen de surcos. E1 MF3 tiene la misma morfología posiblemente que tenía el MF2, pero algo mayor, carácter que $H$. rondoniensis sp. nov. comparte con todas las especies de Pampatheriidae. El alvéolo de MF4 es tiene aspecto reniforme, indicando que el molariforme tenia el lóbulo anterior más ancho que el posterior, y orientado oblicuamente con respecto a la serie dentaria (con el lóbulo posterior en posición labial); en $S$. hondaensis es casi bilobulado y en $K$. paranense de igual forma que el MF3, pero de mayor tamaño; $H$. occidentalis tiene un principio de bilobulación, pero menos marcada que en S. hondaensis. A su vez, en H. majus el MF4 presenta una forma bilobulada con los lóbulos opuestos asimétricos; una morfología semejante se observa en $H$. paulacoutoi, cuyo MF4 se orienta diagonalmente a la serie dentaria en relación con su imbricación (Figuras 4C,D). En H. rondoniensis sp. nov. el alvéolo de MF5 es muy parecido al MF4, reniforme, pero con el lóbulo posterior mayor que el anterior. $K$. paranense presenta MF5 bilobulado, con el lóbulo posterior mayor. $H$. occidentalis, H. majus y H. septentrionalis presentan el MF5 perfectamente bilobulado; en $H$. paulacoutoi se esboza un pequeño tercer lóbulo labial. Los alvéolos de MF6-7 y molariforme MF8 son muy similares entre sí, salvo por el largo, que disminuye progresivamente de adelante hacia atrás, así como por la lobulación más manifesta en la misma dirección. En H. rondoniensis sp. nov. tan solo esos tres molariformes son bilobulados; el alvéolo de MF6 posee una forma bilobulada asimétrica; los de MF7 y MF8 son bilobulados con una estrangulación mediana equidistante del lóbulo anterior y posterior. El MF8 de S. hondaensis es también bilobulado; en $K$. paranense, los MF6 y MF7 presentan un tercer lóbulo, más definido en el último diente, y
Tabla 2. Medidas dentarias: longitud antero-posterior $(\mathrm{mm})$ de $\mathbf{A}$, H. rondoniensis sp. nov., MERO-P-002; B, H. occidentalis, ROM 3881; C, H. majus, UZM 2314; D, H. paulacoutoi, MCL-501/01; E, H. septentrionalis, HMNS 173; F, Pampatherium typum, MACN Pv 11543; G, P. humboldtii, MHD-P-28. Abreviaturas: en el texto.

Table 2. Dental measurements: antero-posterior length $(\mathrm{mm})$ of $\mathbf{A}$, Holmesina rondoniensis sp. nov., MERO-P-002; B, H. occidentalis, ROM 3881; C, H. majus, UZM 2314; D, H. paulacoutoi, MCL-501/01; E, H. septentrionalis, HMNS 173; F, Pampatherium typum, MACN PV 11543; G, P. humboldtii, MHD-P-28. Abbreviations: see text.

\begin{tabular}{cccccccc}
\hline & A & B & C & D & E & F & G \\
\hline MF1 & 8.1 & 10 & 8.1 & - & 10 & 7.8 & 9.2 \\
MF2 & 12 & 13 & 11 & 12 & 12 & 8.2 & 8 \\
MF3 & 17.5 & 16 & 13 & 19 & 14.2 & 9.6 & 10 \\
MF4 & 19 & 17.1 & 19.1 & 20.4 & 17.2 & 15.8 & 14.4 \\
MF5 & 24 & 25 & 24.1 & 26.4 & 21.2 & 16.9 & 21 \\
MF6 & 27.0 & 27.9 & 26 & 26.0 & 23.2 & 19.4 & 23.7 \\
MF7 & 26.5 & 26 & 24.1 & 26.2 & 23.5 & 17.5 & 24.2 \\
MF8 & 21.0 & 23.5 & 22 & 22.8 & 21.2 & 15.4 & 21.7 \\
MF9 & 17.1 & 14.5 & 18 & 15 & 13.2 & 17.9 & 20 \\
\hline
\end{tabular}

el MF8 es bilobulado con una fuerte estrangulación mediana; el MF6 de $H$. occidentalis es muy parecido al alvéolo de $H$. rondoniensis sp. nov., la diferencia consiste en que los lóbulos son más definidos; el MF7 de dicha especie es prácticamente igual al anterior, y el MF8 con un pequeño tercer lóbulo. El MF6 de $H$. majus es más angosto que el MF5; el MF7 en $H$. paulacoutoi, $H$. majus y $H$. septentrionalis muestra un tercer lóbulo intermedio, más pronunciado en H. majus; el MF8 en H. majus es bilobulado, con los lóbulos más redondeados que en $H$. paulacoutoi y menos que en $H$. septentrionalis. Finalmente, el alvéolo de MF9 en $H$. rondoniensis sp. nov. es mucho más simple que los de MF5-MF8 indicando que el diente tenia apenas un ligero surco labial. En cambio, estos dientes en $S$. hondaensis y $K$. paranensis son bilobulados. $H$. majus presenta una vez más un tercer lóbulo, mientras $H$. occidentalis, $H$. majus, $H$. paulacoutoi y $H$. septentrionalis que son bilobulados y prácticamente idénticos.

\section{DISCUSIÓN}

Los pampatéridos, durante el lapso Mioceno tardío-Holoceno temprano, experimentan una moderada diversificación (al menos cinco géneros), como ocurre hasta cierto punto con los gliptodontes, y a diferencia de la diversidad mucho mayor alcanzada por los dasipódidos. Tanto pampatéridos como gliptodontes conservan muchas características morfológicas básicas, pues siguen sendas líneas estructurales muy homogéneas (Vizcaíno et al., 1998; Zurita, 2007a). Estudios paleobiológicos cráneo-mandibulares y dentarios sugieren que, además de una diferenciación morfológica, también existió una diferenciación ecológica entre Pampatherium y Holmesina (Vizcaíno et al., 1998; De Iuliis et al., 2000).

El género Pampatherium parece haber estado adaptado básicamente a consumir vegetación más abrasiva, propia de climas áridos y fríos, en tanto que Holmesina podría haber estado mejor adaptado a ambientes más húmedos y cálidos (Vizcaíno 
et al., 1998; De Iuliis et al., 2000; Scillato-Yané et al., 2005).

En el territorio de la Argentina, Pampatherium typum está registrada desde el Ensenadense (Scillato-Yané et al., 1995), por ejemplo en la ciudad de Buenos Aires y alrededores ("Toscas" del Río de La Plata), asociado con taxones de origen intertropical (Tapiridae y Procyonidae) que sugieren temperaturas relativamente cálidas y húmedas, probablemente relacionadas con los eventos entre Jaramilo y Olduvai, que coinciden con los episodios isotópicos 35-37 y 41-45, o la transgresión Interensenadense (Cione et al., 2002). Otra asociación con $P$. typum está en la base del Lujanense, donde las condiciones ambientales eran probablemente más templadas y un tanto húmedas durante el episodio isotópico OIS 5e (Scillato-Yané et al., 2005). Sin embargo, en el Lujanense tardío de la región pampeana $P$. typum está asociado con una fauna del Último Máximo Glacial. Esta última asociación se verifica de manera similar en el sur de Uruguay, donde $P$. typum ha sido registrado en depósitos del Pleistoceno tardío (Formación Dolores) asociado a varios taxones de mamíferos que actualmente viven en la región patagónica y central de Argentina, y que sugieren al menos condiciones áridas (Ubilla et al., 2009). En el Brasil, de acuerdo con Rizzoto \& Oliveira (2005), fueron colectados sedimentos organógenos y restos de madera en dos niveles fosilíferos de las localidades de Abunã y Taquara; los niveles fosilíferos de esas dos localidades corresponde al mismo nivel de la localidad Araras/Periquitos, donde se han encontrado la mayoría de los fósiles cuaternarios de Rondônia, entre ellos el holotipo de Holmesina rondoniensis sp. nov. El análisis palinológico de esas dos localidades indica una predominancia de elementos vegetales típicos de un bosque con características húmedas y cálidas (Rizzoto \& Oliveira, 2005); dichos elementos apuntan a una transición entre el Pleniglacial y el Interglacial (base del estadio isotópico 4e), con temperaturas más cálidas y húmedas que aquellas del Lujanense tardío de la Provincia de Buenos Aires, el cual es posterior al Interglacial o estadio isotópico OIS 5e. En resumen, se reafirma la hipótesis de Vizcaíno et al. (1998), De Iuliis et al. (2000) y Scillato-Yané et al. (2005) que proponen condiciones más cálidas y húmedas para las especies de Holmesina, mientras que las de Pampatherium estarían sujetas a climas más fríos y condiciones más áridas o semiáridas.

El registro de especies de los dos géneros cuaternarios de pampatéridos ha sido comprobado en Rio Grande do Sul, sur de Brasil, en dos localidades distintas: Arroio Chuí y Ponte Velha I, en las formaciones Santa Vitória (Sistema Barreira/ Laguna III) y Formación Touro Passo, respectivamente. En la primera localidad están registrados Pampatherium typum y Holmesina paulacoutoi (Oliveira et al., 2003; Kerber \& Oliveira, 2008; Oliveira \& Pereira, 2009b) y en la segunda P. typum, $P$. humboldtii y H. paulacoutoi probablemente de la misma unidad (Oliveira \& Pereira, 2009b). Oliveira \& Kerber (2009a) observan que la mayoría de los depósitos pleistocenos de Rio Grande do Sul (e.g. Formación Touro Passo) son de origen fluvial, lo cual dificulta establecer una correlación precisa con las edades Bonaerense y Lujanense de la región pampeana (sensu Cione \& Tonni, 1999, 2005). En este contexto, resulta factible que algunas de las especies de pampatéridos procedentes de una misma localidad no hayan sido sincrónicas. Nos hallaríamos ante una mezcla de taxones con distintos requerimientos ecológicos e indicativos de distintos ambientes, intertropicales en algunos casos $(P$. humboldtii y $H$. paulacoutoi) y pampeanos en otros (P.typum).

La simpatría (no la sincronía) de los pampatéridos en el territorio argentino es conocida en la Mesopotamia argentina, donde Pampatherium typum y Holmesina paulacoutoi han sido exhumados en la Formación Toropí (Provincia de Corrientes, Scillato-Yané et al., 2005; Rodríguez-Bualó et al., 2009). Recientes dataciones por luminiscencia indican que esos sedimentos habrían sido depositados en el Lujanense tardío (ca. 50-35 Ka; OIS 3). Según Tonni (2009), este período presenta cambios climático-ambientales muy complejos, con pulsos que van desde lapsos cálidos, por un lado, a fríos y áridos o semiáridos, por el otro. Estas oscilaciones podrían explicar la presencia en un mismo yacimiento de ambos taxones (P. typum y H. paulacoutoi) sin que ellos sean sincrónicos (Carlini et al., 2008a). Esta situación se repetiría en la Formación Fortín Tres Pozos (Provincia de Formosa) donde se han registrado P. typum y H. paulacoutoi; la Formación Fortín Tres Pozos es correlacionable con las formaciones Toropí y Yupoí (Rodríguez-Bualó et al. 2009).

La distribución de los dos géneros de pampatéridos pleistocenos puede ser el resultado de un patrón alopátrico provocado por procesos de especiación durante la última parte del Pleistoceno (al menos para las especies de Holmesina en Sudamérica). Durante este lapso, los cambios climáticos asociados con la transición entre el Último Interglacial y el Último Máximo Glacial, habrían promovido el surgimiento de barreras infranqueables para estas especies (Clapperton, 1993; Van der Hammen \& Absy, 1994; Haberle \& Maslin, 1999). Probablemente una especie generalizada de "Holmesina like", o incluso de Kraglievichia, se haya dispersado hacia América del Norte y se haya diferenciado como Holmesina septentrionalis (Scillato-Yané et al., 2005; Carlini et al., 2008b; Carlini \& Zurita, 2010). El segundo evento de dispersión habría ocurrido en el Pleistoceno con Pampatherium, y tuvo como consecuencia la diferenciación de $P$. mexicanum Edmund, 1996, la única especie del género en América del Norte (Edmund, 1996; Scillato-Yané et al., 2005). Por último, tuvo lugar un evento de reingresión de Holmesina desde América del Norte hacia América del Sur, y luego la diferenciación de las especies sudamericanas: $H$. rondonienis sp. nov, $H$. occidentalis, $H$. majus y $H$. paulacoutoi.

Se reconocen para América del Sur seis especies y dos géneros ampliamente distribuidos: Holmesina occidentalis, $H$. rondoniensis sp. nov., H. majus, H. paulacoutoi, Pampatherium typum y P. humboldtii (Figura 5). H. rondoniensis sp. nov. se registra hasta el momento en Rondônia (Brasil), Amazonia sudoccidental. H. occidentalis se distribuye en Ecuador, Perú, Venezuela y Colombia (Edmund, 1996; Scillato-Yané et al., 2005). En Ecuador, es conocida para la Provincia de Guaya (Península de Santa Helena; Hofstetter, 1952); en Perú, se registra en Cuspinique y Desierto de Talara (Marshall et al., 1984). En el norte de Venezuela, se encuentra en los estados de Falcón (Royo \& Gómez, 1960; Rincón, 2004; Rincón \& 


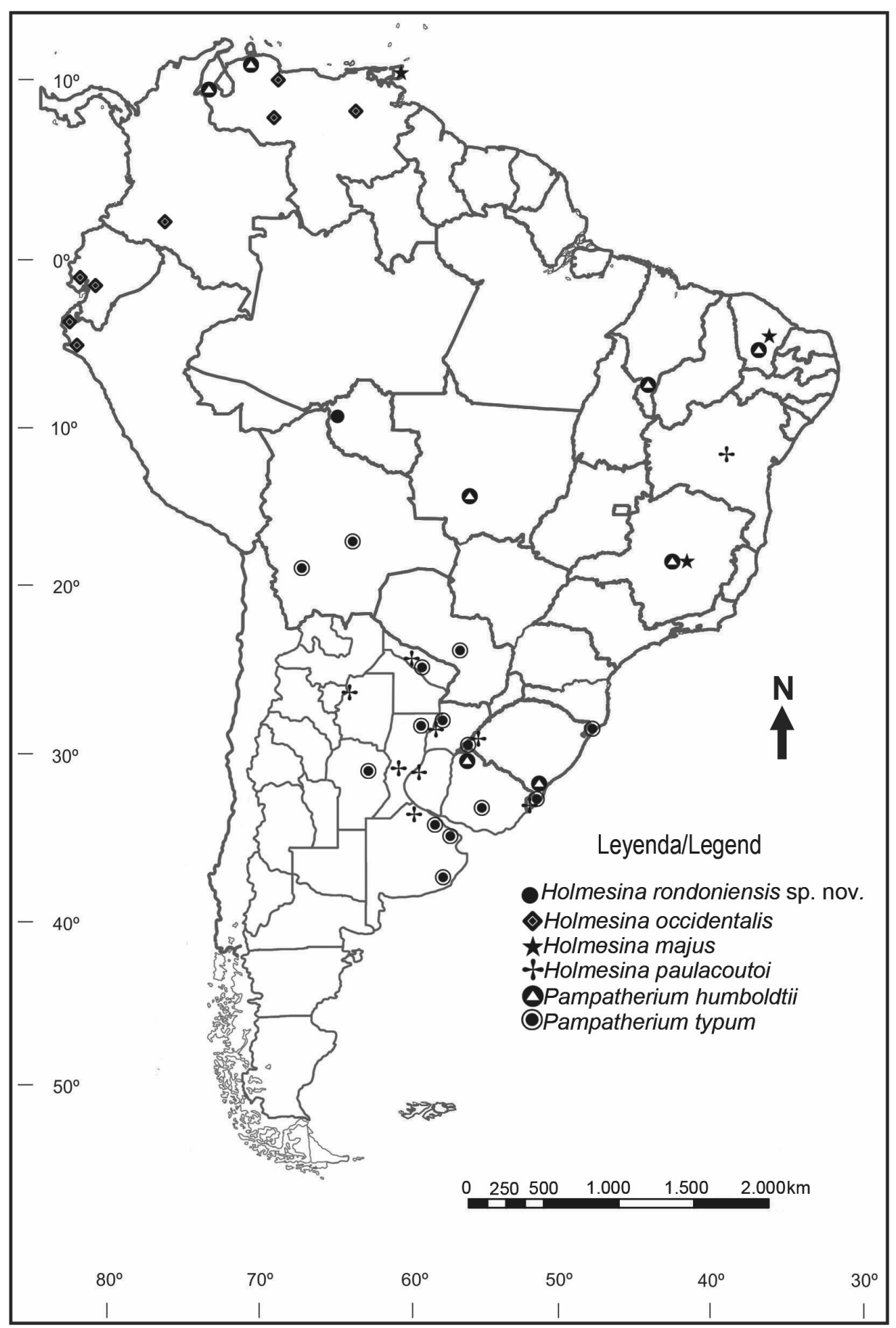

Figura 5. Distribución de las especies de pampatéridos cuaternarios de América del Sur. Ver referencias bibliográficas en el texto.

Figure 5. Distribution of South American Quaternary pampatheres species. See text for references.

White, 2007), Carabobo (Marshall et al., 1984), Cojedes (Rincón et al., 2009) y Monagas (Rincón et al., 2009); en Colombia está registrada en el Departamento de Huila (Edmund, 1996). H. majus está distribuida en Brasil, en los estados de Ceará, Minas Gerais (Scillato-Yané et al., 2005; Oliveira \& Pereira, 2009b); además, en Venezuela, Trinidad. Oliveira \& Pereira (2009b) descartan la presencia de $H$. majus para Rio Grande do Sul, mencionada previamente por Edmund (1996) y Scillato-Yané et al. (2005). H. paulacoutoi se distribuye en Brasil en los estados de Bahia (Cartelle \&
Bohórquez, 1985; Cartelle, 1999), Rio Grande do Sul (Kerber \& Oliveira, 2008; Oliveira \& Kerber, 2009a; Oliveira et al., 2003; Oliveira \& Pereira, 2009b) y Rio Grande do Norte (Porpino et al., 2004). En Argentina, posee una distribución que abarca las provincias de Corrientes (Scillato-Yané et al., 2005), Santa Fe (Scillato-Yané et al., 2005), Entre Ríos (Scillato-Yané et al., 2005), Buenos Aires (Scillato-Yané et al., 2005); asimismo, recientemente para Formosa (RodríguezBualó et al. 2009) y Santiago del Estero (Chimento \& Agnolin, 2011). 
Pampatherium typum está registrado en Paraguay, Bolivia, Brasil, Uruguay y Argentina. En Paraguay se registra cerca de Asunción (Carlini \& Tonni, 2000). En Bolivia, se conoce en Ñuapúa (Hoffstetter, 1968a) y Tarija (Hoffstetter, 1963). En Brasil, en los estados de Santa Catarina y Rio Grande do Sul (Paula Couto, 1980; Kerber \& Oliveira, 2008; Oliveira \& Kerber, 2009a; Oliviera \& Pereira, 2009b). En Uruguay, en el Departamento de Canelones (Ubilla et al., 2009). En Argentina, se registra en Buenos Aires, Santa Fe, Córdoba, Formosa y Corrientes (Scillato-Yané, 1980; Scillato-Yané et al., 2005). P. humboldtii se halla en Brasil, Venezuela y Uruguay. En Brasil en los estados de Mato Grosso, Minas Gerais, Ceará, Rio Grande do Sul (Lund, 1840; Winge, 1915; Edmund, 1996; Oliveira \& Pereira, 2009b). En Venezuela fue reconocida para el estado de Zulia (Rincón et al., 2008) y en Uruguay para el Departamento de Artigas (Ubilla \& Perea, 1999).

De todas las especies comparadas sólo Holmesina occidentalis se asemeja en rasgos generales a la nueva especie, pero se distingue de ella no sólo por su tamaño, sino por su morfología propia. Es probable que la similitud entre ambas especies esté relacionada con su proximidad temporal y geográfica de diferenciación, ya que restos de $H$. occidentalis están registrados en Colombia, Ecuador, Perú y Venezuela, y $H$. rondoniensis sp. nov. está registrado más al sur de la Amazonia, en Brasil. En síntesis, H. rondoniensis sp. nov. se diferencia del resto de las especies de Holmesina por una serie de caracteres, entre ellos: cráneo más grácil y estilizado, nasales muy estrechos y rectos, con el ancho distal algo menor que el proximal; región frontal menos convexa y más corta; parietal largo y estrecho con la cresta sagital más estrecha y más alta; en dicha región los canales y forámenes vasculares son reducidos; cresta lambdoidea muy expandida hacía atrás; arcada cigomática larga y grácil, menos elevada dorsoventralmente (en la región mediana) y sin el proceso infraorbitario; paladar más estrecho; alvéolo de MF1 muy próximo al margen anterior del paladar, imbricación dentaria entre alvéolos de MF2, MF3 y MF4, espacio interalveolar muy reducido; apófisis pterigoides delgadas y poco rugosas, en posición oblicua; supraoccipital alto, estrecho y escavado a ambos lados de la cresta occipital, la cual es larga y prominente.

Holmesina rondoniensis sp. nov. y H. occidentalis muestran rasgos más generalizados que las restantes especies del género. La primera es más pequeña y menos robusta. De acuerdo con Vizcaíno et al. (1998) y De Iuliis et al. (2000), mediante a la morfología del cráneo y mandíbula, es posible proponer interpretaciones paleobiológicas y eventuales relaciones filogenéticas. Estos autores hipotetizan que Vassalia maxima estaba adaptada a consumir vegetales más abrasivos por poseer una serie de características que permiten tal condición, tales como: una osteodentina más resistente (en Holmesina, la osteodentina es más débil o incluso puede estar ausente; ver De Iuliis \& Edmund, 2002), cresta sagital muy prominente, arcada cigomática robusta y proceso infraorbitario largo y rugoso. Según Vizcaíno et al. (1998) y De Iuliis et al. (2000), H. occidentalis estaba adaptada a consumir vegetales más blandos que las demás especies hasta entonces conocidas de Holmesina; mencionan que $H$. paulacoutoi poseía adaptaciones que sugieren el consumo de vegetales algo más duros que $H$. occidentalis, pero algo más blandos que Pampatherium. En consecuencia, es factible suponer que $H$. rondoniensis sp. nov., menos robusta que $H$. occidentalis y mucho menos que las demás especies del género, sería una especie adaptada al consumo de vegetales más blandos que lo inferido para $H$. occidentalis. Las características craneanas que permiten reforzar tal afirmación son: (i) región parietal más angosta, poco rugosa, con pocos canales y reducidos forámenes vasculares, lo cual implicaría un menor desarrollo de la musculatura temporal; (ii) arcada cigomática sin la típica elevación dorsoventral y sin el proceso infraorbitario, caracteres que sugieren una menor acción muscular del masetero; y (iii) apófisis pterigoides delgadas y pocos rugosas, orientadas oblicuamente y muy distintas de aquellas de las demás especies de Holmesina, las cuales presentan apófisis pterigoides grandes y gruesas, notablemente rugosas y proyectadas lateralmente.

\section{CONCLUSIONES}

Se propone en este trabajo una nueva especie para el género Holmesina, $H$. rondoniensis sp. nov., siendo esta proveniente de la Formación Rio Madeira (Pleistoceno tardío-Holoceno), Estado de Rondônia, Brasil. Esta es la quinta especie del género para el Pleistoceno y la cuarta especie para Sudamérica. $H$. rondoniensis sp. nov. sería la especie más estilizada de todos los pampatéridos pleistocenos e incrementa la diversidad conocida para Holmesina, hasta entonces con las especies pleistocenas, $H$. majus, H. occidentalis, H. paulacoutoi y H. septentrionalis y una pliocena, H. floridanus. Finalmente, es sugerido que en la región Amazónica en general, las condiciones fitogeográficas y climáticas serían más propicias para las especies de Holmesina que para las de Pampatherium.

\section{AGRADECIMIENTOS}

Al A. Ranzi, quien por primera vez mencionó a uno de los autores (F.G.L.) la presencia del cráneo objeto del estudio. Al entonces secretario L.C. Venceslau, de la Secretaria da Cultura, Esporte e Lazer (Secel) do Estado de Rondônia, por su significativo apoyo a las investigaciones paleontológicas. A la N. Silva, responsable de la colección paleontológica del Museu do Estado de Rondônia, por su amabilísima disposición. Al C. Cartelle, del Museu de Ciências Biológicas da Pontifícia Universidade Católica de Minas Gerais, por las generosas informaciones sobre los Pampatheriidae y al acceso de los materiales. A A. Kramarz y J.C. Fernicola, del Museo Argentino de Ciencias Naturales "Bernadino Rivadavia", y al M.A. Reguero y al L. Pomi, de la Facultad de Ciencias Naturales y Museo de La Plata, por su ayuda para acceder a las colecciones. Al J. Soloviy, del Museo Histórico Departamental de Artigas, por su atenciosa disponibilidad. A la entonces curadora T. Hätten del Universitets Zoologisk Museum, por su cordialidad en facilitar el acceso de los materiales. Al L.R. González-Ruiz y al E.T. Bostelmann, por el envío de algunas fotografías. Al E.P. Tonni, por su valiosa colaboración 
sobre algunos temas de gran importancia para el desarrollo de este trabajo. A la A. Francia, por el envío de bibliografías e importantes informaciones sobre las formaciones Toropí y Yupoí. Al E.O.C. Aponte por la reconstrucción de Holmesina utilizado en el mapa e informaciones de los registros de pampaterios en Venezuela. A la M. Castro, por la colaboración en la traducción al inglés de algunos fragmentos. Al A.E. Zurita y al revisor anónimo por sus valiosos y objetivos comentarios, que permitieron mejorar este trabajo.

\section{REFERENCIAS}

Abrantes, E.A.L. \& Bergqvist, L.P. 2006. Propuesta filogenética para los Dasypodidae (Mammalia: Cingulata). In: V. Gallo; P.M. Brito; H.M.A. Silva. \& F.J. Figueiredo (eds.) Paleontologia de Vertebrados: Grandes Temas e Contribuições Científicas, Interciência, 1:261-274.

Adamy, A. \& Pereira, L.A.C. 1991. Projeto Ouro e Gemas - Frente Rondônia. Relatório Anual, Companhia de Pesquisa de Recursos Minerais, p. 8-35.

Adamy, A. \& Romanini, S.J. 1990. Geologia da região Porto VelhoAbunã. Folhas Porto Velho (SC 20-V-B-V), Mutumparaná (SC 20-V-C-VI), Jaciparaná (SC 20-V-D-I), Abunã (SC.20-V-C-V), estados de Rondônia e Amazonas. Programa de levantamentos geológicos básicos do Brasil, DNPM/CPRM, Brasília, 237 p.

Ameghino, F. 1883a. Sobre una colección de mamíferos fósiles del piso mesopotámico de la formación patagónica recogidos por el Prof. Pedro Scalabrini. Boletín de la Academia Nacional de Ciencias de Córdoba, 5:101-116.

Ameghino, F. 1883b. Sobre una nueva colección de mamíferos fósiles recogidos por el Profesor Pedro Scalabrini en las barrancas del Paraná. Boletín de la Academia Nacional de Ciencias de Córdoba, 5:257-306.

Ameghino, F. 1914. Notas sobre algunos fósiles nuevos de la formación pampeana. In: A.J. Torcelli (ed.) Obras Completas y Correspondencia Cientifica de Florentino Ameghino. La Plata, 2:13-17.

Bordas, A.F. 1939. Craniometría y regíon auditiva de "Chlamydotherium typum" Ameghino. Physis, Revista de la Sociedad Argentina de Ciencias Naturales, 14:447-460.

Bronn, H.G. 1838. Abbildungen und Beschreibungen der für die Geobirgs-Formationen bezeichnendsten Versteinerugen. Lethea Geognostica, 2:545-1350.

Carlini, A.A. \& Scillato-Yané, G.J. 1993. Origin and evolution of the "Glyptodontoids". Journal of Vertebrate Palaeontology, 3:28A.

Carlini, A.A. \& Tonni, E.P. 2000. Mamíferos Fósiles del Paraguay. Cooperación Técnica Paraguayo-Alemana, Proyecto Sistema Ambiental del Chaco, Proyecto Sistema Ambiental Región Oriental, Buenos Aires, 108 p.

Carlini, A.A. \& Zurita, A.E. 2010. An introduction to cingulate evolution and their evolucionary history during the Great American Biotic Interchange: biogeographical clues from Venezuela. In: M.R.Sánchez-Villagra; O.A. Anguilera \& A.A. Carlini (eds.) Urumaco \& Venezuelan Paleontology, Indiana University Press, p. 233-255.

Carlini, A.A.; Zurita, A.E. \& Miño-Bolini, A.R. 2008a. Reseña paleobiogeográfica de los Xenarthra (Mammalia) del Pleistoceno tardío de la region Mesopotámica (Argentina). Revista del Instituto Superior de Correlación Geológica, INSUGEO, 17:259-270.

Carlini, A.A.; Zurita, A.E. \& Orangel, A.A. 2008b. North American glyptodontines (Xenarhra, Mammalia) in the Upper Pleistocene of South America. Paläontologische Zeitschriff, 82:125-138.
Cartelle, C. 1999. Pleistocene mammals of the Cerrado and Caatinga of Brazil. In: J.F. Eisenberg \& K.H. Redford (eds.) Mammals of the Neotropics, The University of Chicago Press, p. 27-46.

Cartelle, C. \& Bohórquez, G.A. 1985. Pampatherium paulacoutoi, uma nova espécie de tatu gigante da Bahia, Brasil (Edentata, Dasypodidae). Revista Brasileira de Zoologia, 2:229-254.

Carranza-Castañeda, O. \& Miller, W.E. 2004. Late tertiary terrestrial mammals from central Mexico and their relationship to South American immigrants. Revista Brasileira de Paleontologia, 7:249-261.

Castellanos, A.1927. Breve notas sobre los Clamidoterios. Publicación del Centro Estudiantes de Ingeniería de Rosario, p. 1-8.

Castellanos, A.1937. Anotaciones sobre la línea filogenética de los Clamiterios. Publicación Instituto Fisiografia y Geologia, Universidad Nacional Litoral, 26:1-47.

Chimento, N.R. \& Agnolin, F.L. 2011. Mamíferos del pleistoceno superior de Santiago del Estero (Argentina) y sus afinidades paleobiogeográficas. Papeis Avulsos de Zoologia, 51:83-100. doi:10.1590/S0031-10492011000600001

Cione, A.L. \& Tonni, E.P. 1999. Bioestratigraphy and chronological scale of upper-most Cenozoic in the Pampean Area, Argentina. Quaternary of South America and Antarctic Peninsula, 12:23-51.

Cione, A.L. \& Tonni, E.P. 2005. Bioestratigrafía basada em mamíferos del Cenozóico Superior de la Província de Buenos Aires, Argentina. In: R.E. Barrio; R.O. Etcheverry; M.F. Caballé \& E. Llambias (eds.) Geología y recursos minerales de la Província de Buenos Aires, p. 183-200.

Cione, A.L.; Tonni, E.P. \& San Cristóbal, J. 2002. A MiddlePleistocene marine transgression in central-eastern Argentina. Current Research in the Pleistocene, 19:16-18.

Clapperton, C.M. 1993. Nature of environmental change in South America at the Last Glacial Maximum. Palaeogeographic, Palaeoclimatology and Palaeoecology 101:189-208.

De Iuliis, G.; Bargo, S.M. \& Vizcaíno, S.F. 2000. Variation in skull morphology and mastication in the fossil giant Armadillos Pampatherium spp. and allied genera (Mammalia: Xenarthra: Pampatheriidae), with comments on their systematics and distribution. Journal of Vertebrate Paleontology, 20:743-754.

De Iuliis, G. \& Edmund, A.G. 2002. Vassalia maxima Castellanos, 1946 (Mammalia: Xenarthra: Pampatheriidae), from Puerta del Corral Quemado (Late Miocene to Early Pliocene), Catamarca Province, Argentina. In: R.J. Emry (ed.) Cenozoic Mammals of Land and Sea. Tributes to the career of Clayton E. Ray, Smithsonian Contributions to Paleobiology, 93:49-64.

Downing, K.F. \& White, R.S. 1995. The cingulates (Xenarthra) of the Leisey Shell Pit Local Fauna (Irvingtonian), Hisllsborough Country, Florida. Bulletin of the Florida Museum of Natural History, 37:375-396.

Edmund, A.G. 1985a. The armor of fossil giant armadillos (Pampatheriidae, Xenarthra, Mammalian). Pearce-Sellards Series, Texas Memorial Museum, 40:1-20.

Edmund, A.G. 1985b. The fossil giant armadillos North America (Pampatheriinae, Xenarthra=Edentata). In: G.G. Montgomery (ed.) The Evolution and Ecology of Armadillos, Sloths, and Vermilinguas, Smithsonian Institution Press, p. 83-94.

Edmund, A.G. 1987. Evolution of the genus Holmesina (Pampatheriidae, Mamalia) in Florida, with remarks on taxonomy and distribution. Pearce-Sellards Series, Texas Memorial Museum, 45:1-20.

Edmund, A.G. 1996. A review of Pleistocene giant armadillos (Mammalia, Xenarthra, Pampatheriidae). In: K.M. Stewart \& K.L. Seymor (eds.) Paleoecology and palaeoenvironments of late Cenozoic mammals, University of Toronto Press, p. 300-321. 
Edmund, A.G. \& Theodor, J. 1997. A new giant Armadillo. In: R.F. Kay; R.L. Cifelli, J.J. Flynn \& R. Madden (eds.) Vertebrate Paleontology of the Miocene Honda Group, Republic of Colombia, Smithsonian Institution Press, 14:227-232.

Engelmann, G.F. 1985. The phylogeny of the Xenarthra. In: Montgomery, G.G. (ed.) The Evolution and Ecology of Armadillos, Sloths, and Vermilinguas, Smithsonian Institution Press, p. 51-64.

Flynn, J.J.; Kowals, B.J.; Nuñes, C.; Carranza-Castañeda, O.; Miller, W.E.; Swisher, III C.C. \& Lindsay, E.H.2005. Geochronology of Hemphillian-Blancan age strata, Guanajuato, Mexico, and implications fot timing of the Great American Biotic Interchange: Journal Geology, 113:287-307.

Gaudin, T. J. \& Wible, J. R. 2006. The phylogeny of living and extinct armadillos (Mammalia, Xenarthra, Cingulata): a craniodental analysis. In: M.T. Carrano,; T.J Gaudin,; R.W. Blob \& J.R. Wible (eds.) Amniote Paleobiology: Perspectives on the Evolution of Mammals, Birds and Reptiles, University of Chicago Press, p. 153-198.

Gervais, H. \& Ameghino, F.1880. Les mammifères fossiles de l'Ámérique du Sud. Paris-Buenos Aires, Savy-Ignon H., 225 p.

Góis, F.L. 2005. Estudo descritivo e geométrico dos Cingulata (Mammalia, Xenarthra) do Neógeno e Quaternário da Amazônia Sul-Ocidental. Curso de Ciências Biológicas, Universidade Federal de Rondônia, Monografia de Bacharelado, $58 \mathrm{p}$.

Góis, F.; Nascimento, E.R.; Porto, A.S.; Holanda, E.C. \& Cozzuol, M.A. 2004. Ocorrências de Cingulata dos gêneros Kraglievichia e Holmesina do Terciário e Quaternário da Amazônia SulOcidental. Ameghiniana, 41:49R.

Góis, F.L. \& Scillato-Yané, G.J. 2008. Un novedoso pampatérido (Xenarthra, Glyptodontoidea, Pampatheriidae) del Pleistoceno de Rondônia, sudoeste de la Amazonia, Brasil. In: CONGRESO LATINOAMERICANO DE PALEONTOLOGÍA DE VERTEBRADOS, 3, 2008. Resúmenes, Neuquén, p. 113.

Góis, F.L.; González Ruiz, L.R. \& Scillato-Yané, G.J. 2010. Uso de la morfometría geométrica en la comparación morfológica craneana en Cingulata (Xenarthra): implicaciones evolutivas y funcionales. Ameghiniana, 47:13R.

Haberle, S.G. \& Maslin, M.A. 1999. Late Quaternary vegetation and climatic change in the Amazon basin based in A-50,000 years pollen record from the Amazon fan, PDP site 932. Quaternary Research, 51:27-38.

Hill, R.V. 2005. Integrative morphological data sets for phylogenetic analysis of Amniota: the importance of integumentary characters and increased taxonomic sampling. Systematic Biology, 54:530547. doi: 10.1080/10635150590950326

Hill, R.V. 2006. Comparative anatomy and histology of xenarthran osteoderms. Journal of Morphology, 267:1441-1460. doi: 10.1002/jmor. 10490

Hoffstetter, R. 1952. Les mammifères pleistocenes de la République de 1'Équateur. Mémoires Societé Géologique de France, 66:1391.

Hoffstetter, R. 1953. Sur la présence d'um tatou géant du genre Holmesina dans le Pléistocene de l'Équateur (Amerique du Sud). Compte Rendu Sommaire des séances de la Société Géologique de France, 6:101-102.

Hoffstetter, R., 1958. Xenarthra. In: Piveteau, J. (ed.) Traité de Paléontologie, Masson, p. 535-636.

Hoffstetter, R. 1963. La faune pléistocène de Tarija (Bolivie). Nota préliminaire. Bulletin Museum National d'Histoire Naturelle, 35:194-203.
Hoffstetter, R. 1968. Ñuapúa, un gisement de vertébrés pléistocènes dans le Chaco bolivien. Bulletin Museum National d'Histoire Naturelle, 40:823-836.

Holanda, E.C. \& Cozzuol, M.A. 2006. Análise morfométrica de dentários de Tapirus (Mammalia, Perissodactyla) do Pleistoceno superior da Amazônia Sul-Ocidental, Brasil. Paleontologia em Destaque, p. 60.

Holanda, E.C.; Ferigolo, J. \& Ribeiro, A.M. 2011. New Tapirus species (Mammalia: Perissodactyla: Tapiridae) from the upper Pleistocene of Amazonia, Brazil. Journal of Mammalogy, 92:111-120. doi: 10.1644/10-MAMM-A-144.1

James, G.T. 1957. An edentate from the Pleistocene of Texas. Journal of Paleontology, 31:797-808.

Kerber, L. \& Oliveira É.V. 2008. Fósseis de vertebrados da Formação Touro Passo (Pleistoceno Superior), Rio Grande do Sul, Brasil: atualização dos dados e novas contribuições, Gaea, 4:49-64. doi:10.4013/gaea.2008.2.02

Leidy, J. 1889a. Description of the vertebrate remains from Peace Creek, Florida. Transactions of the Wagner Free Institution of Science of Philadelphia, 2:19-32.

Leidy, J. 1889b. Fossil vertebrates from Florida. Production Academy Natural Science Philadelphia, p. 96-97.

Lund, P. 1839. Blik paa Brasiliens Dyreverden för Sidste Jordomvaeltning. Anden Afhandling: Pattedyrene. Det Kongelige Danske Videnskabernes Selskbas Naturvidenskabelige og Mathematiske Afhandlinger, 8:61-144.

Lund, P. 1840. Blikpaa Brasiliens Dyreverden för Sidste Jordomvaeltning. Tredie Afhandling: Forsaettelse af Pattedyrene. Det Kongelige Danske Videnskabernes SelskbasNaturvidenskabelige og Mathematiske Afhandlinger, 8:217-272.

Marshall, L.G.; Berta, A.; Hoffstetter, R.; Pascual, R.; Reig, O.A.; Bombin, A. \& Mones, A. 1984. Mammals and stratigraphy: geochronology of the continental mammal-bearing Quaternary of South American. Palaeovertebrata, Mémoire Extrardinaire p. 1-76.

Mckenna, M.C. \& Bell, S.K. 1997. Classification of Mammals. Above the Species Level. New York, Columbia University Press, 640p.

Mead, J.I. ; Swift, S.L. ; White, R.S. ; McDonald, H.G. \& Báez, A. 2007. Late Pleistocene (Rancholabrean) Glyptodont and Pampathere (Xenarthra, Cingulata) from Sonora, México. Revista Mexicana de Ciencias Geológicas, 24:439-449.

Mones, A. 1986. Nomen Dubium vs. Nomen Vanum. Journal of Vertebrate Palaeontology, 9:232-234.

Mones, A. 1994. Los silencios de Florentino Ameghino. Problemas de la bibliografía, sistemática y nomenclatura. Senckenbergina Biologica, 74:9-20

Morgan, G.S. \& Hulbert, R.C.Jr. 1995. Overview of the geology and vertébrate paleontology of the Leisey Shell Pit Local Fauna, Hillsborough Country, Florida. Bulletin Florida Museum Natural History, 37:1-92.

Nascimento, E.R.; Holanda, E.C.; Góis, F.L. \& Cozzuol, M. A. 2005. Inferências paleoambientais para os depósitos fossilíferos do Quaternário da Amazônia Sul-Ocidental, Brasil. In: CONGRESSO BRASILEIRO DE PALEONTOLOGIA, 19/ CONGRESSO LATINOAMERICANODE PALEONTOLOGIA, 6, 2005. Resumos, Aracaju, SBP, CD-ROM.

Oersted, H.C. 1939. Oversigt over det kongelike Danslce Vidensleabernes selsicabs Forhandlinger og dets Medlemmers Arbeiler $i$ Aaret 1838, Conpenhagen, p. 5-14.

Oliveira, É.V.; Faccin, J.R. \& Pereira, J.C. 2003. O pampatério Holmesina (Mammalia, Pampatheriidae) no Quaternário do Rio Grande do Sul. Ameghiniana, 40:64R. 
Oliveira, É.V. \& Kerber, L. 2009a. Paleontologia e aspectos geológicos das sucessões do final do Neógeno no sudoeste do Rio Grande do Sul, Brasil. Gaea, 5:21-3. doi: 10.4013/ gaea.2009.51.03

Oliveira, É.V. \& Pereira, J.C. 2009b. Intertropical cingulates (Mammalia, Xenarthra) from the Quaternary of Southern Brazil: systematics and paleobiogeographical aspects. Revista Brasileira de Paleontologia, 12:167-178. doi:10.4072/rbp.2009.3.01

Patterson, B. 1967. Order Edentata in the fossil record, Mammalia. London Geology Society, p. 771-772.

Patterson, B. \& Pascual, R. 1968. Evolution of mammals on southern continents. Quarterly Review of Biology, 43:409-451.

Patterson, B. \& Pascual, R. 1972. The fossil mammal fauna of South America. In: A. Keast; C. Erk \& B. Glass (eds.) Evolution, Mammals and Southern Continents, State University New York Press, p. 247-309.

Patterson, B.; Segall, W. \& Turnbull, D.W. 1989. The ear region Xenarthrans (= Edentata: Mammalia) Part I. Cingulates. Fieldiana Geology, 1-46.

Paula Couto, C. 1954. Sobre um gliptodonte do Uruguai e um tatu fóssil do Brasil. Notas preliminares e estudos. Rio de Janeiro, Departamento Nacional de Produção Mineral, Divisão de Geologia e Mineralogia, 10 p. (Boletim 80)

Paula Couto, C. 1979. Tratado de Paleomastozoologia. Rio de Janeiro, Academia Brasileira de Ciências, 590 p.

Paula Couto, C. 1980. Um tatu gigante do Pleistoceno de Santa Catarina. Anais da Academia Brasileira de Ciências, 52:527-531.

Pennington, R.T.; Prado, D.E. \& Pendry, C.A. 2000. Neotropical Seasonallydry forest and Quaternary vegetation changes. Journal of Biogeography, 27:217-233.

Porto, A.S.; Góis, F.; Nascimento, E.R.; Holanda, E.C. \& Cozzuol, M.A. 2004. Xenarthra Pilosa (Edentata) do Quaternário do Estado de Rondônia. Ameghiniana, 41:60R.

Radambrasil. 1978. Levantamento de Recursos Naturais. Folha SC 20 Porto Velho. Rio de Janeiro, DNPM, 663 p.

Rincón, A.D. 2004. Los mamíferos fósiles del Pleistoceno de la Cueva del Zumbador (Fa. 116) estado Falcón, Venezuela. Boletín de la Sociedad Venezolana de Espeleología, 37:18-25.

Rincón, A.D.; Parra, G.E.; Prevosti F.J.; Alberdi, M.T. \& Bell, C.J. 2009. A preliminary assessment of the mammalian fauna from the Pliocene-Pleistocene el Breal de Orocual locality, Monagas State, Venezuela. Museum of Northern Arizona Bulletin, 64:593620.

Rincón, A.D. \& White, R. 2007. Los Xenarthra Cingulata del Pleistoceno tardío (Lujanense) de Cerro Misión, Estado Falcón, Venezuela. Boletín de la Sociedad Venezolana de Espeleología, 41:2-12.

Rincón, A.D.; White, R.S. \& McDonald, H.G. 2008. Late Pleistocene cingulates (Mammalia: Xenarthra) from Mene de Inciarte Tar Pits, Sierra de Perijá, Western Venezuela. Journal of Vertebrate Paleontology, 28:197-207. doi: 10.1671/0272-4634(2008)028[0677:TLSCTX]2.0.CO;2

Rizzotto, G.J. 2005. Projeto Rio Madeira. Levantamento de informações para subsidiar o estudo de viabilidade do aproveitamento hidrelétrico (AHE) do Rio Madeira. AHE: Jirau, Porto Velho. Relatório Final, CPRM, Serviço Geológico do Brasil, 213 p.

Rizzotto, G.J.; Quadros, M.L.E.S. \& Castro, J.M.R. 2007. Geologia. In: M.L.E.S. Quadros \& G.J. Rizzotto (eds.) Geologia e Recursos Minerais do Estado de Rondônia: sistema de informações geograficas-SIG: texto explicativo do mapa geológico e de recursos minerais do estado de Rondônia, CPRM, Serviço Geológico do Brasil, p. 12-67.
Rodríguez-Bualó, S.; Zurita, A.E.; Carlini, A.A.; Friederich, J. 2009. Los Pampatheriidae (Mammalia, Xenarthra, Cingulata) del norte del norte de la Argentina: taxonomía y paleobiogeografía. In: COMUNICACIONES CIENTÍFICAS Y TECNOLÓGICAS, 2009. Resúmenes, Universidad Nacional del Noroeste, versión digital, www. unne.edu.ar.

Royo \& Gómez, V. 1960. El yacimiento de vertebrados pleistocenos de Muaco, Estado Falcón, Venezuela, con industria lítica humana. In: INTERNATIONAL GEOLOGICAL CONGRESS, 1960. Reports of the twenty-first session, 21:154-157.

Sant'Anna Filho, M.J. \& Vidal, D.A. 1999. Ocorrência de Mixotoxodon larensis (Toxodontidae, Mammalia) em sedimentos do paleoleito do rio Madeira no Estado de Rondônia, Brasil. In: CONGRESSO BRASILEIRO DE PALEONTOLOGIA, 16. Resumos, Crato, SBP, p. 38.

Schobbenhaus, C. 1984. Geologia do Brasil. Brasília, DNPM, 501p.

Scillato-Yané, G.J. 1982. Los Dasypodidae (Mammalia, Edentata) del Plioceno y Pleistoceno de Argentina. Facultad de Ciencias Naturales y Museo, Universidad Nacional de La Plata, Tesis doctoral, $244 \mathrm{p}$.

Scillato-Yané, G.J.; Carlini, A.A.; Tonni, E.P. \& Noriega, J.I. 2005. Paleobiogeography of the late Pleistocene pampatheres of South America. Journal of South American Earth Sciences, 20:132138. doi:10.1016/j.jsames.2005.06.012

Scillato-Yané, G.J.; Carlini, A.A.; Vizcaíno, S.F. \& Ortiz Jaureguizar, E. 1995. Los Xenarthros. In: M.T. Alberdi; G. Leone \& E.P. Tonni (eds.) Evolución biológica y climática de la región Pampeana durante los últimos cinco millones de años. Un ensayo de correlación con el Mediterráneo occidental, Museo de Ciencias Naturales, Consejo de Investigaciones, 12:183-209.

Sellards, E.J. 1915. Chlamytherium septentrionalis, an edentate from the Pleistocene of Florida. American Journal Science 4:19-31.

Simpson, G.G. 1930. Holmesina septentrionalis, extinct giant armadillo de Florida. American Museum Novitates, 422:1-10.

Stehli, F.G. \& Webb, S.D. 1985. The Great American Biotic Interchange. New York. Plenum Press, $532 \mathrm{p}$.

Tonni, E.P. 2009. Los mamíferos del Cuaternario de la Región Pampeana de Buenos Aires, Argentina. In: A.M. Ribeiro; S.G. Bauermann \& C.S. Scherer (eds.) Quaternário do Rio Grande do Sul, integrando conhecimentos, Monografias da Sociedade Brasileira de Paleontologia, p. 2-14.

Ubilla, M. 1996. Paleozoología del Cuaternario continental de la cuenca norte del Uruguay: biogeografía, cronología y aspectos climático ambientales. Universidad de la República Uruguay, Tesis Doctoral, $232 \mathrm{p}$.

Ubilla, M. \& Perea, D. 1999. Quaternary vertebrates of Uruguay: a biostratigraphic, biogeographic and climatic overview. Quaternary of South America and Antarctic Penninsula, 12:7590.

Ubilla, M.; Perea, D.; Rinderknecht, A. \& Corona A. 2009. Pleistocene mammals from Uruguay: biostratigraphic, biogeographic and environmental connotations. In: A.M. Ribeiro; S.G. Bauermann \& C.S Scherer (eds.) Quaternario do Rio Grande do Sul, integrando conhecimentos, Monografías da Sociedade Brasileira de Paleontología, p. 217-230.

Van der Hammen, T. \& Absy, M.L. 1994. Amazonia during the last glacial. Palaeogeographic, Palaeoclimatology and Palaecology, 109:247-261.

Vizcaíno, S.F. 2009. The teeth of the "toothless": novelties and key innovations in the evolution of xenarthrans (Mammalia, Xenarthra). Paleobiology, 35:343-366. doi:10.1666/ 0948373/09/3503-0003 
Vizcaíno, S.F.; De Iuliis, G. \& Bargo, S.M. 1998. Skull shape, mastigatory apparatus, and diet of Vassalia and Holmesina (Mammalia: Xenarthra: Pampatheriidae): when anatomy constrains destiny. Journal of Mammalian Evolution, 5:291322.

Winge, H. 1915. Hordfunde og nulevende Gumlere (Edentata) fra Lagoa Santa, Minas Geraes, Brasilien. Med Udsigt over Gumlernes indbyrdes Slaegtskab, Copenhagen. E. Museo Lundii, 3:1-321.

Winge, H. 1941. Edentates (Edentata). In: A.S. Jensen; R. Spärck \& H. Volsoe (eds.) The Interrelationships of the Mammalian Genera, Reitzels Forlag, p. 319-341.

Woodburne, M.O. 2010. The Great American Biotic Interchange: dispersals, tectonics, climate, sea level and holding pens. Journal
Mammalian Evolution, 17:245-264. doi:10.1007/s10914-0109144-8

Zurita, A.E. 2007. Sistemática y evolución de los Hoplophorini (Xenarthra, Glyptodontidae, Hoplophorinae. Mioceno tardío-Holoceno temprano). Importancia bioestratigráfica, paleobiogeográfica y paleoambiental. Universidad Nacional de La Plata, Tesis doctoral, $367 \mathrm{p}$.

Zurita, A.E.; Scillato-Yané, G.J. \& Mones, A. 2007. Aspectos nomenclaturales de la Familia Glyptodontidae (Mammalia, Xenarthra): el caso de Sclerocalyptus Ameghino, Hoplophorus Lund y la Tribu Sclerocalyptini. Ameghiniana, 44:241-244. doi: AMGHB2-0002-7014/07

Received in August, 2011; accepted in June, 2012. 